\title{
Computational Studies on Acquisition and Adaptation of Ocular Following Responses Based on Cerebellar Synaptic Plasticity
}

\author{
KENJI YAMAMOTO, ${ }^{1,2}$ YASUSHI KOBAYASHI, ${ }^{3}$ AYA TAKEMURA, ${ }^{2,4}$ KENJI KAWANO,,${ }^{2,4}$ \\ AND MITSUO KAWATO ${ }^{5}$ \\ ${ }^{1}$ Japan Science and Technology Corporation; ${ }^{2}$ Neuroscience Research Institute, National Institute of Advanced Industrial \\ Science and Technology, Ibaraki 305-8568; ${ }^{3}$ National Institute for Physiological Sciences, Aichi 444-8585; ${ }^{4}$ Core Research \\ for Evolutional Science and Technology, Japan Science and Technology Corporation, Ibaraki 305-8568; and ${ }^{5}$ ATR Human \\ Information Science Laboratory, Kyoto 619-0288, Japan
}

Received 28 February 2001; accepted in final form 2 November 2001

Yamamoto, Kenji, Yasushi Kobayashi, Aya Takemura, Kenji Kawano, and Mitsuo Kawato. Computational studies on acquisition and adaptation of ocular following responses based on cerebellar synaptic plasticity. J Neurophysiol 87: 1554-1571, 2002; 10.1152/jn. 00166.2001. To investigate how cerebellar synaptic plasticity guides the acquisition and adaptation of ocular following response (OFR), a large-scale network model was developed. The model includes the cerebral medial superior temporal area (MST), Purkinje cells (P cells) of the ventral paraflocculus, the accessory optic and climbing fiber systems, the brain stem oculomotor network, and the oculomotor plant. The model reconstructed temporal profiles of both firing patterns of MST neurons and P cells and eye movements. Model MST neurons $(n=1,080)$ were set to be driven by retinal error and exhibited 12 preferred directions, 30 preferred velocities, and 3 firing waveforms. Correspondingly, each model $\mathrm{P}$ cell contained 1,080 excitatory synapses from granule cell axons (GCA) and 1,080 inhibitory synapses. P cells $(n=40)$ were classified into four groups by their laterality (hemisphere) and by preferred directions of their climbing fiber inputs (CF) (contralateral or upward). The brain stem neural circuit and the oculomotor plant were modeled on the work of Yamamoto et al. The initial synaptic weights on the P cells were set randomly. At the beginning, $\mathrm{P}$ cell simple spikes were not well modulated by visual motion, and the eye was moved only slightly by the accessory optic system. The synaptic weights were updated according to integral-differential equation models of physiologically demonstrated synaptic plasticity: long-term depression and long-term potentiation for GCA synapses and rebound potentiation for inhibitory synapses. We assumed that maximum plasticity was induced when GCA inputs preceded CF inputs by $200 \mathrm{~ms}$. After more than 10,000 presentations of ramp-step visual motion, the strengths of both the excitatory and inhibitory synapses were modified. Subsequently, the simple spike responses became well developed, and ordinary OFRs were acquired. The preferred directions of simple spikes became the opposite of those of CFs. Although the model MST neurons were set to possess a wide variety of firing characteristics, the model $\mathrm{P}$ cells acquired only downward or ipsilateral preferred directions, high preferred velocities and stereotypical firing waveforms. Therefore the drastic transition of the neural representation from the population codes in the MST to the firing-rate codes of simple spikes were learned at the GCA-P cell synapses and inhibitory cells-P cell synapses. Furthermore, the model successfully reproduced the gain- and directional-adaptation of OFR, which was demonstrated by manipulating the velocity and direction of visual motion, respectively. When

Address for reprint requests: K. Yamamoto, Neuroscience Research Institute, AIST, Central 2, 1-1-1, Umezono, Tsukuba, Ibaraki 305-8568, Japan (E-mail: k.yamamoto@aist.go.jp). we assumed that synaptic plasticity could only occur if CF inputs preceded GCA inputs, the ordinary OFR were acquired but neither the gain-adaptation nor the directional adaptation could be reproduced.

\section{IN T R O D U C T I O N}

It is widely accepted that the cerebellum plays an important role in motor learning and adaptation for a wide range of behaviors. Humans and animals with severe cerebellar lesions cannot adequately learn new movements (Baizer and Glickstein 1974; Baizer et al. 1999; Gauthier et al. 1979; Ito et al. 1979; Lisberger et al. 1984; McElligott et al. 1998; Michnovicz and Bennett 1987; Nagao 1983; Pastor et al. 1994; Robinson 1976; Sanes et al. 1990; Thach et al. 1992; Weiner et al. 1983). Cerebellar Purkinje cells ( $\mathrm{P}$ cells), the only output neurons of the cerebellar cortex, receive three major synaptic inputs: a large number of granule-cell axon (GCA) inputs, multiple inhibitory cell (IC) inputs, and a single climbing fiber (CF) input. ICs receive GCA inputs and project their axons to $\mathrm{P}$ cells. P cells exhibit two kinds of spikes: simple spikes (SS) induced by GCA inputs and complex spikes (CS) induced by $\mathrm{CF}$ inputs. The physiologically demonstrated synaptic plasticity of $\mathrm{P}$ cells has been suggested to be the cellular mechanism responsible for movement adaptation and learning. The known types of synaptic plasticity include long-term depression (LTD) and long-term potentiation (LTP) for excitatory GCA synapses, and rebound potentiation (RP) for inhibitory synapses from ICs. LTD results in a decrease in the synaptic efficacy of GCA synapses and is induced by the conjunctive stimulation of GCAs and CFs (Ito and Kano 1982; Ito et al. 1982). It has been shown that LTP is induced presynaptically by stimulation of GCA in the absence of CF stimulation (Hirano 1990; Sakurai 1987). RP results in an increase in the synaptic efficacy of IC synapses and is induced by conjunctive activation of IC and P cells (Kano 1996; Kano et al. 1992). Changes in $\mathrm{P}$ cell firing that explain behavioral changes during adaptation have been recorded for both SSs and CSs (Dufosse et al. 1978; Gilbert and Thach 1977; Nagao 1989; Watanabe 1985). Here, GCA and IC inputs are generally assumed to

\footnotetext{
The costs of publication of this article were defrayed in part by the payment of page charges. The article must therefore be hereby marked "advertisement" in accordance with 18 U.S.C. Section 1734 solely to indicate this fact.
} 
convey the sensory-motor context to P cells. On the other hand, the $\mathrm{CF}$ input is assumed to carry error signals essential for learning and adaptation (Kitazawa et al. 1998; Kobayashi et al. 1998).

The cellular mechanisms of LTD have been intensively studied, mainly in slice and culture preparations (reviewed in Crepel et al. 1996). Based on these in vitro studies, molecular and genetic techniques have been introduced to examine relationships between LTD and behavioral adaptation. For example, the injection of LTD inhibitors into the cerebellum has been found to abolish behavioral adaptation (Li et al. 1995; Nagao and Ito 1991; Yanagihara and Kondo 1996). Furthermore, transgenic mice with protein kinase $\mathrm{C}$ inhibitors expressed in P cells have been found to lack both LTD and motor learning capability (De Zeeuw et al. 1998).

Although there have been extensive experimental studies on $\mathrm{P}$ cell plasticity and its possible roles in motor learning, detailed and realistic computational studies that quantitatively reconstruct the temporal dynamics of both $\mathrm{P}$ cell firing and movement kinematics during motor learning are scarce. However, such computational studies are essential for understanding the mechanisms underlying how these types of synaptic plasticity achieve motor learning and adaptation.

The objectives of this study were to construct a detailed and quantitative neural circuit model that could reproduce both the control and adaptation of ocular following responses and to investigate the above mechanisms. Specifically, we addressed the following six questions. The first question is whether in vivo motor adaptation can be fully explained by the characteristics of $\mathrm{P}$ cell plasticity revealed by in vitro slice and culture preparation studies. The second question concerns whether the time difference between GCA inputs and CF inputs during the induction of LTD is crucial for reproducing behavioral modification. In slice experiments, the amount of change induced in the synaptic efficacy was found to depend on the time interval between CF and GCA stimulations (Chen and Thompson 1995; Karachot et al. 1994). We call this the temporal window of LTD. The temporal window reported by Karachot et al. (1994) was criticized for not being appropriate for motor learning (De Schutter 1995; Lukashin 1996). The third question is whether all reported types of plasticity, i.e., LTD, LTP, and RP, are essential for motor learning (cf. De Shutter 1995). The fourth question concerns the range of motor adaptation and learning, which might be explained by synaptic plasticity in the cerebellar cortex. There are several types of motor learning and adaptation, such as the acquisition of new behaviors, and gain and kinematic adaptation to preexisting movements. The fifth question concerns the learning acquisition of temporal waveforms of SS firing. What proportion of the SS firing characteristics of an individual $\mathrm{P}$ cell is determined by the characteristics of the $\mathrm{CF}$ input to that $\mathrm{P}$ cell through synaptic plasticity? The last question is the most abstract and at the highest level; what does the cerebellar cortex computationally acquire in motor learning with these classes of synaptic plasticity?

To answer these questions adequately, a model must be constructed for a movement whose neural control circuit has been well studied. Second, the model must quantitatively reconstruct SS and CS firings based on experimental data. If the motor control system contains feedback loops, movement kinematics must also be quantitatively reproduced based on experimental data by a model containing such feedback loops.
Previous simulation studies have examined the adaptation and learning of several kinds of movements, such as vestibular ocular reflexes, saccadic eye movements, smooth pursuit, and arm movements in relation to cerebellar synaptic plasticity (Fujita 1982; Gomi and Kawato 1992; Kettner et al. 1997; Raymond and Lisberger 1998; Schweighofer et al. 1996, 1998). Unfortunately, some of the preceding prerequisites were not satisfied in these studies.

Ocular following responses (OFRs) are movements for which we can construct a simulation model that satisfies all of the above requirements. OFRs are reflex eye movements induced with short latencies by motion of a large-field visual stimulus. They exhibit several types of behavioral adaptation (Miles and Kawano 1986). Previous physiological studies have revealed that the medial superior temporal area (MST) of the cerebral cortex, the dorsolateral pontine nucleus (DLPN), and the ventral paraflocculus (VPFL) of the cerebellum are important loci for controlling OFR (reviewed in Kawano 1999). The characteristics of the neural activity in the MST (Kawano et al. 1994), DLPN (Kawano et al. 1992), and VPFL (Gomi et al. 1998; Kawano and Shidara 1993; Kobayashi et al. 1998; Shidara and Kawano 1993; Shidara et al. 1993) during OFR have been very intensively studied. In particular, SS firing waveforms have been reconstructed by inverse dynamics models of eye movements (Gomi et al. 1998; Shidara et al. 1993). We have already developed a quantitative model of the preceding neural network that controls OFR as a feedback control system (Yamamoto et al. 1997, 2000). Kobayashi et al. (1998) have also developed a quantitative model of CS firing waveforms based on the physiological data.

Based on these previous studies, a large-scale network model for two-dimensional OFR was developed, and its longterm development and short-term adaptation were simulated. With this realistic and quantitative model, we were able to examine the preceding questions about the computational roles of synaptic plasticity in motor learning.

\section{MODEL}

\section{Ocular following responses: behavioral and neurophysiological studies}

Behavioral studies of monkeys and humans have shown that sudden movements of a visual scene evoke short-latency OFRs (Gellman et al. 1990; Miles et al. 1986; reviewed in Kawano 1999). The experimental paradigm used to study OFRs is described in the following text (Kawano et al. 1992). A random dot pattern in a full visual field positioned in front of a monkey is suddenly moved upward, downward, leftward, or rightward in a velocity-step, position-ramp manner. We call this kind of stimulus a ramp stimulus. The visual field starts to move 50-300 ms after the end of a saccadic eye movement directed toward the central part of the screen $\left( \pm 10^{\circ}\right)$. The ramp movement of the stimulus usually lasts 150 or $300 \mathrm{~ms}$ before a mechanical shutter shuts off the visual field. The eyes of the monkey start to follow the stimulus about $50 \mathrm{~ms}$ after the ramp stimulus onset (Miles et al. 1986).

OFRs undergo two types of adaptation: gain adaptation after repeated "speed-step" stimuli, and directional adaptation after repeated "direction-step" stimuli (Miles and Kawano 1986). The "speed-step" stimuli contain a "step-up stimulus" and a 
"step-down stimulus." For the step-up stimulus, the monkey is given a ramp stimulus slower than $100 \%$ for the first $150 \mathrm{~ms}$. The velocity of the stimulus is changed to $100^{\circ}$ s for the next $150 \mathrm{~ms}$. For the step-down stimulus, the monkey is given a ramp stimulus slower than $100 \%$ for the first $150 \mathrm{~ms}$. The velocity of the stimulus is changed to $0 \%$ for the next $150 \mathrm{~ms}$. After repeating the step-up trials, the OFR gain of the monkey, which is defined as the maximum eye velocity divided by the stimulus velocity, increases adaptively for the test ramp stimuli at the initial speed. After repeating the step-down trials, the OFR gain of the monkey decreases for the test ramp stimuli. In the direction-step trials, the direction of the stimulus is changed to $90^{\circ}$ counterclockwise $150 \mathrm{~ms}$ after the onset of the ramp stimulus. After repeated direction-step trials, the OFR direction is changed to the counterclockwise direction for the test ramp stimuli in the initial direction.

Many previous studies have investigated neural firing during OFR in several brain loci, as shown in Fig. 1. Movement of the retinal image induces neural activity in the visual cortex, the MST (Kawano et al. 1994), and the DLPN (Boussaoud et al. 1992; Brodal 1978; Glickstein et al. 1980, 1985; Kawano et al. 1992; Maunsell and van Essen 1983; May and Andersen 1986; Tusa and Ungerleider 1988; Ungerleider et al. 1984). The axons of DLPN neurons project to the cerebellar VPFL (Glickstein et al. 1990; Langer et al. 1985; Shidara and Kawano 1993) as mossy fibers (Kawano and Shidara 1993) and innervate granule cells in the VPFL cortex. The axons of granule cells (GCAs) project partly to the P cells of the VPFL as parallel fibers (PFs). These GCAs transmit excitatory firing stimuli to P cells. GCAs also project to ICs, i.e., stellate cells and basket cells, in the molecular layer of the cerebellar cortex. ICs then inhibit P cells when they are activated. The inputs of GCAs and ICs to P cells modulate the SSs of the P cells. The $\mathrm{P}$ cells of the VPFL project to the brain stem and influence firing of extraocular motor neurons, thus moving the eyes. Retinal image motion information is also conveyed to the accessory optic tract and to the inferior olivary nucleus. Cell firing in the inferior olivary nucleus is transmitted to $\mathrm{P}$ cells by CFs. The CF inputs induce CSs of P cells. It has been suggested that the indirect pathway for slow eye movements (Fuchs and Mustari 1993; Mustari et al. 1994), which contains the accessory optic tract, might control OFRs in addition to the MST-DLPN-VPFL pathway (Inoue et al. 2000). Lesions in the VPFL abolish a large portion of OFRs (Miles et al. 1986), suggesting that the MST-DLPN-VPFL pathway is the major control system for OFRs.

The direction, speed, and temporal characteristics of cell firing differ markedly between the mossy fiber inputs and the SS firing outputs of the VPFL (Kawano 1999; Kawato 1999; Takemura et al. 2001). First, the preferred direction changes from a uniform distribution to the horizontal and vertical axes only. Each cell in the MST (Kawano et al. 1994) and DLPN (Kawano et al. 1992) exhibits a truncated cosine-function direction tuning in its firing response to large-field visual motion, and its preferred direction is distributed in wide range of $360^{\circ}$ without anisotropy. Similar characteristics were reported on the firings of mossy fibers (Kawano and Shidara 1993). However, for vertical P cells, the direction tuning curves are well fitted by full cosine functions, and the preferred direction of CS responses is upward and that of SS responses is downward (Kobayashi et al. 1998; Shidara and Kawano 1993). For horizontal P cells, the preferred direction of CS responses is contralateral and that of SS responses is ipsilateral. Second, the wide distribution of preferred velocities in VPFL inputs changes to a narrow distribution in VPFL outputs. Each MST cell (Kawano et al. 1994) and DLPN cell (Kawano et al. 1992) exhibits an individual preferred stimulus velocity, which is widely dispersed over $10,20,40,80$, or $160 \%$ s. On the other hand, each P cell has a preferred velocity for SSs only at high velocities, such as 80 or $160 \%$ s (Shidara and Kawano 1993). Finally, a wide variety of phasic and tonic temporal waveforms in the input firings change to the stereotypical SS waveform,

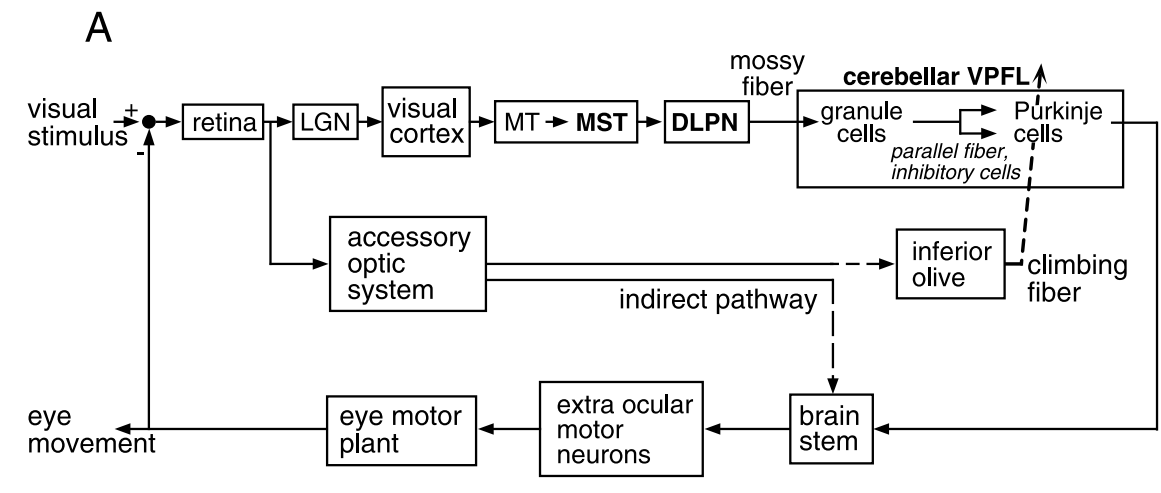

B

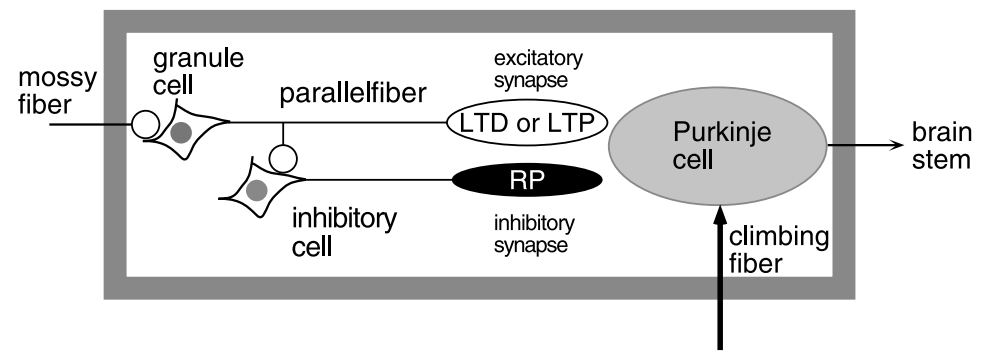

FIG. 1. A: a large scale network model for 2-dimensional ocular following response (OFR). MST, medial superior temporal area; DLPN, dorsolateral pontine nucleus; VPFL, ventral paraflocculus. $B$ : a magnified diagram of the cerebellar VPFL shown in A. LTD, longterm depression; LTP, long-term potentiation; RP, rebound potentiation. 
which is well reconstructed by the inverse dynamics model of eye movements (Gomi et al. 1998; Shidara et al. 1993). Takemura et al. (2001) reconstructed the temporal firing frequency patterns of cells in the MST and DLPN, and SSs in the VPFL, from the acceleration, velocity, and position of eye movements or retinal error. They analyzed the discharges of neurons including open-loop/closed-loop portion without considering the extra-retinal information (efference copy, etc.) and succeeded in quantitatively showing the temporal difference in the neural firing of these areas. They found that the acceleration and velocity coefficients of the MST and DLPN ranged widely, whereas those of the VPFL more compactly.

\section{Previous and present models of OFR control}

Our previous OFR control system model (Yamamoto et al. 1997) quantitatively reproduced SS firing and eye movements. The squared correlation coefficients between simulated and experimental data were 0.81 for SSs and 0.99 for the eye velocity. In that model, the velocity and acceleration of retinal error were first delayed by $39 \mathrm{~ms}$, then saturated, filtered, and finally weighted to become SS firings. These SS firings passed through a filter that was estimated by inverse-dynamics analysis (Gomi et al. 1998; Shidara et al. 1993) and were then delayed by $12 \mathrm{~ms}$ to become the eye movement.

In this paper, we maintain the basic structure of this previous model but extend it to a new model that consists of the following three compartments (Fig. 1A). The first compartment is the MST-DLPN-mossy-fiber-GCA/IC pathway, which is basically the same as in the previous model but is much more biologically detailed and realistic. Figure 2 enlarges the first compartment within the entire system. The second compartment is new and represents the inferior olivary and CF system. The third is also new and represents the accessory optic system. Furthermore, to simulate cerebellar plasticity, we introduce good quantitative models of GCA, IC, and CF firing. In the following text, we explain these three compartments and the synaptic plasticity model.

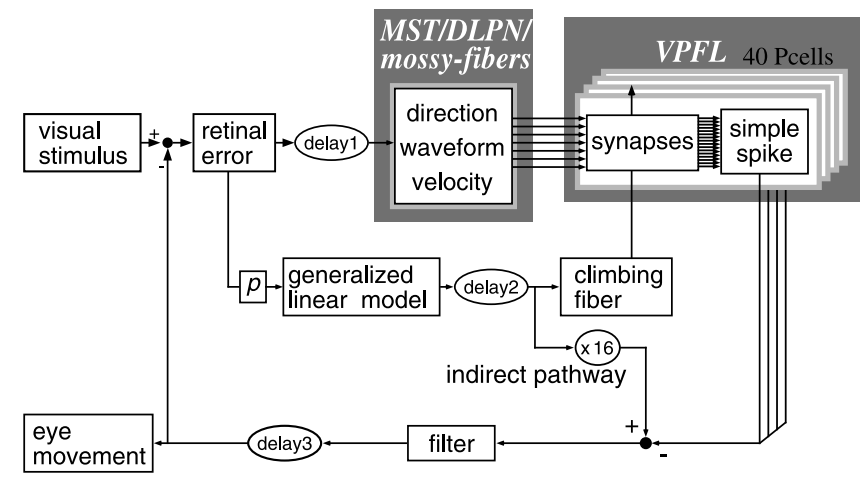

FIG. 2. A detailed diagram of the model, enlarging the medial superior temporal area (MST)-DLPN-VPFL pathway. The model contains 1,080 MST cells and $40 \mathrm{P}$ cells. MST firing is computed from retinal error by making preferred directions (direction), temporal patterns (waveform), and preferred velocities (velocity). The granule cell axons and inhibitory cells carry the MST firing to the $40 \mathrm{P}$ cells through excitatory and inhibitory synapses with individual weights (synapses). The weighted summation of these inputs becomes the SS firing (simple spike). The SS outputs from the VPFL are added to the output from the indirect pathway before the filter to become the eye movement. The retinal error is the visual stimulus motion minus the eye movement. $p$ denotes a Laplace transformation operator.
A
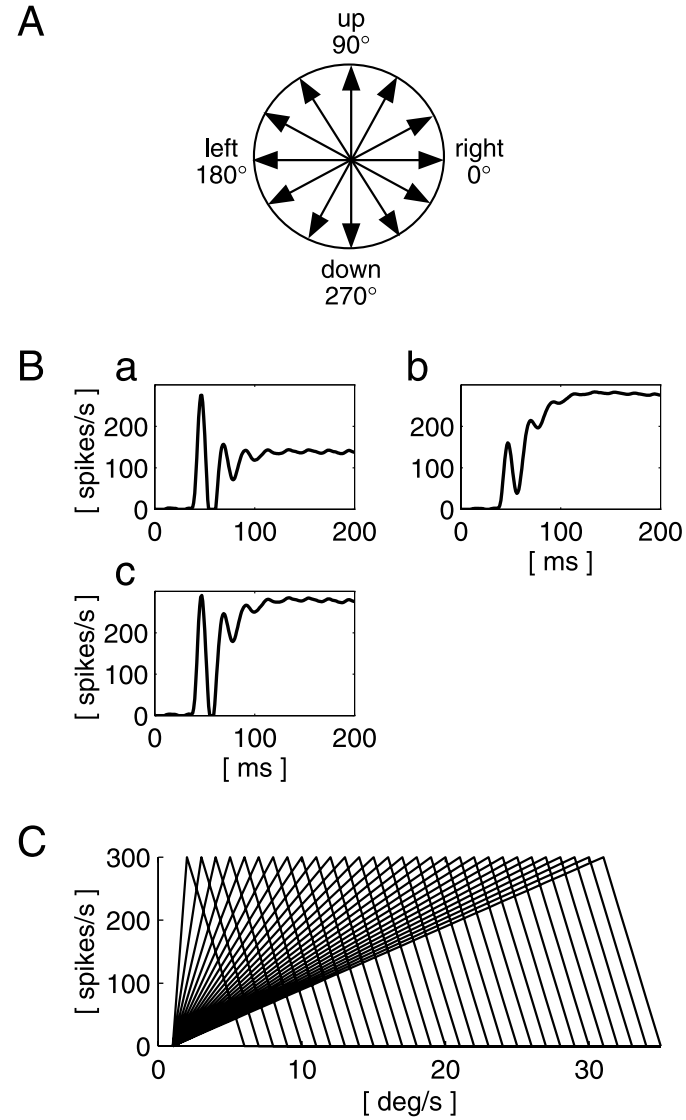

FIG. 3. Directional $(A)$, temporal $(B)$, and velocity $(C)$ tuning characteristics of a model MST firing. A: modeling of the direction selectivity of MST cells. Twelve groups of cells possess their preferred directions every $30^{\circ} ; 0^{\circ}$ is defined as rightward, and the angle is measured in a counterclockwise rotation. $B$ : modeling of the temporal firing patterns of MST cells. The temporal patterns were modeled by summing the acceleration and velocity of the retinal error weighted by 3 kinds of ratios, such that the maximum of the phasic and tonic components of the firing exhibited the three ratios 2:1 (a), 1:2(b), and 1:1 (c). $C$ : modeling the selectivity of MST cells on the stimulus velocity. Thirty curves show saturated firing as a function of stimulus velocity for 30 cells with preferred velocities distributed over $10-300 \%$ s every $10 \%$ from left to right. The abscissa denotes the stimulus velocity. The ordinate denotes the maximal possible firing-frequency output from each MST cell.

\section{Model of MST-DLPN-mossy-fiber-GCA/IC pathway}

The model MST cells in the first compartment are divided into 12 groups according to the 12 preferred directions covering the entire $360^{\circ}$ range separated by $30^{\circ}$ (Fig. $3 A$ ). The directional tuning of MST cell firing is described by the following truncated cosine tuning function

$$
f(\theta)= \begin{cases}f_{\text {max }} \cos \left(\theta-\theta_{\mathrm{P}}\right), & \text { for }\left|\theta-\theta_{\mathrm{P}}\right|<90 \\ 0, & \text { for }\left|\theta-\theta_{\mathrm{P}}\right| \geq 90\end{cases}
$$

Here, $f(\theta), \theta_{\mathrm{P}}$ (degree), $\theta$ (degree), and $f_{\max }$ denote the firing frequency (spikes/s) of the cell, the preferred direction of the cell, the direction of the stimulus, and the maximum firing frequency, respectively. The width of the half-decay for this truncated cosine tuning model is $60^{\circ}$ and agrees well with the experimental data, which show the width of the half-decay as $58^{\circ}$ (Kawano et al. 1994).

To reproduce the experimentally observed dispersion in temporal waveforms of MST cell firings (Takemura et al. 2001), the model MST cells are classified into three groups 
possessing three different temporal firing patterns (Fig. 3B). The firing frequency of each group is reconstructed by adding the filtered accelerations and filtered velocities of the retinal error with the weights $0.005: 0.5,0.0025: 1$, and $0.005: 1$, respectively. We have used the filters of our previous model (Yamamoto et al. 1997): $1 /\left(0.00001 p^{2}+0.0013 p+1\right)$ for the velocity and $1 /\left(0.0001 p^{2}+0.03 p+1\right)$ for the acceleration. Here, $p$ denotes a Laplace transformation operator. The time constants of the filters were selected to reproduce recorded firing patterns from filtered velocity and acceleration of retinal error. The filtered acceleration and velocity contribute to the phasic and the tonic components in the firing waveforms, respectively (Yamamoto et al. 1997). The three firing patterns have their maximum initial phases and tonic phases with ratios of 2:1 (Fig. 3Ba), 1:2 (Fig. 3Bb), and 1:1 (Fig. 3Bc), respectively, when the test ramp stimulus is $10 \%$ s.

Regarding the preferred velocities, the MST cells are divided into 30 groups, covering $10-300 \%$ s every $10 \%$ (Fig. $3 C$ ). This assumed distribution of MST model cell preferred velocities corresponds well with experimentally obtained distributions (Kawano et al. 1994). The MST model cell fires at a maximum of 300 spikes/s for the preferred velocity. While a majority of MST cells with low preferred velocities do not fire at high velocities, many of the MST cells with high preferred velocities fire even at low velocities (A. Takemura, personal communication). To accommodate these experimental observations, we prepared 30 velocity-tuning curves (Fig. 3C), which have long tails for lower velocities than the preferred velocity but short tails for higher velocities for higher velocities. For example, when the ramp stimulus is $80 \%$ s, the model cell with a preferred velocity of $80 \%$ s fires at a maximum of 300 spikes/s. In contrast, the model cell with a preferred velocity of $50 \%$ s fires at a maximum of 75 spikes/s with the same stimulus. The model cell with a preferred velocity of $100 \%$ fires at a maximum of 240 spikes $/ \mathrm{s}$. The ensemble mean of the outputs from these 30 models corresponds well with the experimentally observed ensemble mean of MST cell firings (Kawano et al. 1994).

A total of 1,080 models of MST cells were obtained from 12 groups for preferred direction, 3 groups for different temporal patterns of the firing frequency, and 30 groups for different preferred velocities $(12 \times 3 \times 30$ equals 1,080 models $)$. No remarkable difference has been reported among the firing characteristics of MST cells, DLPN cells, and mossy fibers
(Kawano and Shidara 1993; Kawano et al. 1992, 1994). Accordingly, we used the MST cell firing as the cell firing for both DLPN cells and mossy fibers. In addition, no recording has been attempted from GCAs or ICs during OFR. Therefore the current model assumes that the GCA firing is the same as the mossy fiber firing. The IC firing is assumed to be the same as the GCA firing; therefore the MST-DLPN-mossy-fiber pathway contains 1,080 GCAs and 1,080 ICs, which are connected to $40 \mathrm{P}$ cell models. Therefore $1,080 \times 2 \times 40$ synapses made with a total of $40 \mathrm{P}$ cells.

\section{Model of climbing fiber input}

The preferred direction of CSs in the VPFL is either upward or contralateral, and the direction-tuning curve is well modeled by cosine functions (Kobayashi et al. 1998). Accordingly, the right VPFL of the model contains two types of P cells (vertical and horizontal); the CF inputs of the vertical $\mathrm{P}$ cells prefer the upward stimulus direction, and the CF inputs of the horizontal $P$ cells prefer the leftward direction. The left VPFL also contains two types of $\mathrm{P}$ cells; the CF inputs of the vertical $\mathrm{P}$ cells prefer upward stimuli, and the CF inputs of the horizontal $\mathrm{P}$ cells prefer rightward stimuli. Using these CS preferred directions and their hemispheres, we classify the model $\mathrm{P}$ cells into four groups, two on each side (right h-P cells, right v-P cells, left v-P cells, and left h-P cells from top to bottom in Fig. 4).

To reproduce observed variability in $\mathrm{CF}$ firing characteristics in the temporal domain of individual $\mathrm{P}$ cells experimentally, we prepared 10 different $\mathrm{CF}$ temporal firing patterns for 10 different $\mathrm{P}$ cells in each of the four groups. They were determined by CS recordings from $10 \mathrm{P}$ cells of one monkey reported in Kobayashi et al. (1998). The firing probability of $\mathrm{CF}$ at time $t, \mathrm{CF}(t)$, was well reproduced by a generalized linear model of the acceleration, velocity, and position of the retinal error (Kobayashi et al. 1998). We calculated each deviance for the reconstruction of the temporal CS pattern by the generalized linear models of various combinations of acceleration, velocity, and position of retinal error, and applied the $\chi^{2}$ test to the sets of deviance. As a result, we confirmed that the generalized linear model of only the velocity of the retinal error (retinal slip) was statistically sufficient. Accordingly, we reconstructed the temporal pattern of the CF firing frequency with a generalized linear model by only considering the retinal slip as follows: $\mathrm{CF}(t)=S(B \cdot \dot{r}+C)$.

Here, $S(x)=\exp x /(1+\exp x) \cdot \dot{r}$ denotes either the horizontal

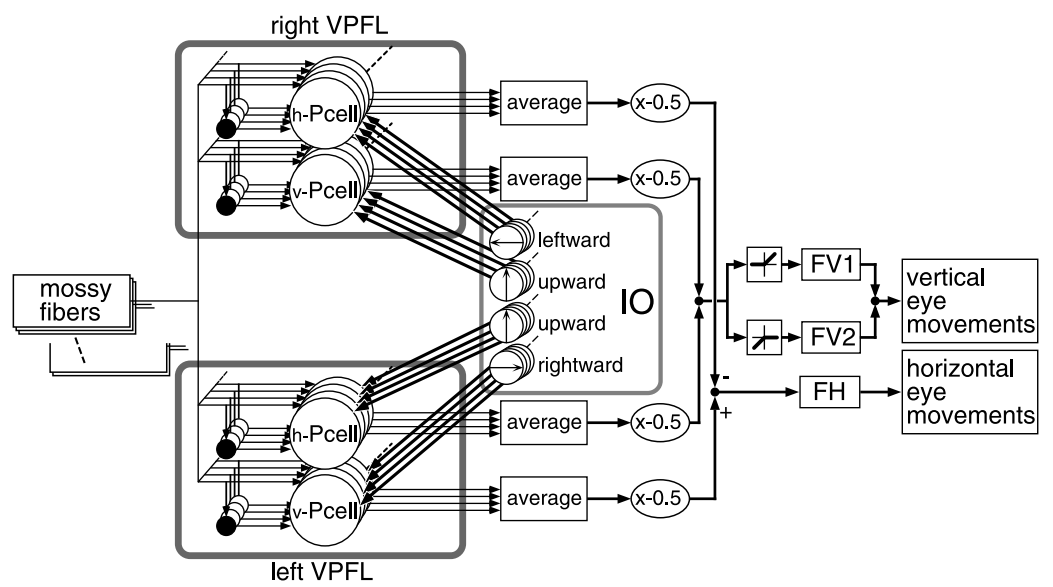

FIG. 4. A detailed diagram of the block of the model that contains $\mathrm{P}$ cells and the downstream control network. $10 \mathrm{P}$ cells within each of the 4 groups (right $h$, right $v$, left $v$, and left $h$ ) receive 10 different climbing fiber (CF) inputs from 10 different inferior olive (IO) cells. The SS outputs from these $40 \mathrm{P}$ cells are first averaged, weighted, and summed or subtracted, and finally filtered differently by the 3 different filters, FV1, FV2, or FH, to generate upward, downward, or horizontal movements. 
or vertical component of the retinal slip. Because the SS firing frequency of most $\mathrm{P}$ cells remains lower than 3 spikes/s (Kobayashi et al. 1998), a CS firing frequency $>3$ spikes/s was reduced to 3 spikes/s. Note that the $\mathrm{CF}$ cosine tuning was reproduced with this equation. We estimated 10 sets of $B, C$ coefficients by using a maximum-likelihood estimation of the recorded data of CS from $10 \mathrm{P}$ cells during an upward $80 \% \mathrm{~s}$ ramp stimulus (Kobayashi et al. 1998). For the $10 \mathrm{CF}$ models within each $\mathrm{P}$ cell group, we used $B$ and $C$ derived from the data. One $\mathrm{P}$ cell model received one $\mathrm{CF}$ model input. Therefore the four types of $\mathrm{P}$ cell groups and 10 types of temporal $\mathrm{CF}$ firing frequencies produced $4 \times 10=40$ different $\mathrm{CF}$ firings and, accordingly, a total of 40 model P cells (see Fig. 4).

\section{Model system of downstream of VPFL}

Here, we describe the model system downstream from the VPFL (Fig. 4). The SS firing of $10 \mathrm{P}$ cells within each of the four groups are first averaged. These averaged outputs from left and right vertical cell groups are further averaged, are reversed in sign, because $\mathrm{P}$ cells are inhibitory neurons, and are finally fed into two filters (FV1 and FV2) to compute the vertical component of a 2-degree-of-freedom eye movement. Here, the upward eye movement is defined as positive. On the other hand, the averaged output from the right horizontal $\mathrm{P}$ cells is sign-reversed and halved and is then subtracted from that of the left horizontal cell group outputs (Fig. 4). This subtraction is fed into the horizontal filter $(\mathrm{FH})$ to produce the horizontal component of the eye movement. Here, the leftward eye movement is defined as positive.

For the filters that represent the combined characteristics of the neural system downstream of the VPFL and the oculomotor plant, we used the filters developed in our previous study (Yamamoto et al. 1997). 1/(0.0442 $\left.p^{2}+2.20 p\right)$ represents FV1 in Fig. 4, when the firing frequency of SSs of the vertical $\mathrm{P}$ cells decreases below the spontaneous firing level of the vertical $\mathrm{P}$ cells. $1 /\left(0.107 p^{2}+2.13 p-3.42\right)$ represents $\mathrm{FV} 2$ in Fig. 4, when the vertical $\mathrm{P}$ cells increase their SS firing frequency. $1 /\left(0.107 p^{2}+2.13 p-3.42\right)$ is FH in Fig. 4 for the horizontal $\mathrm{P}$ cells.

\section{Model of the indirect pathway}

Here, we describe the model compartment of the indirect pathway that parallels the MST-DLPN-VPFL pathway. The MST-DLPN-VPFL pathway for OFR control is known as the direct pathway for the control of optokinetic responses (OKR) (Fuchs and Mustari 1993; Mustari et al. 1994). The OKR control system has another pathway, the indirect pathway, which contains the accessory optic system (Fuchs and Mustari 1993; Mustari et al. 1994) (Fig. 1). The indirect pathway has a velocity storage system and seems to work well several seconds after the onset of the visual stimulus for OKR (reviewed in Fukushima et al. 1992). The visual stimulus for OKR is not very different from that for OFR. Inoue et al. (2000) reported that cells in the nucleus of the optic tract (NOT) in the indirect pathway change their firing frequency during OFR. On the other hand, it is reported that the destruction of the VPFL (a part of the direct pathway) drastically reduces the OFR magnitude (Miles et al. 1986). These reports suggest that the indirect pathway is somewhat involved, but not significantly, in the control of OFR. The firings of the indirect pathway are fed into the IO nucleus, and are then conveyed to P cells by CFs, which in turn cause CSs. Actually, the firing frequency of the indirect pathway is similar in waveform to the firing frequency of CFs, although the firing frequency is much higher (Inoue et al. 2000). We therefore modeled the firing frequency of the indirect pathway by multiplying the firing frequency of CFs by 16 (Fig. 2). This model induces a $1 \%$ s eye velocity with a $10 \%$ stimulus without the MST-DLPN-VPFL pathway. The firing frequency of the indirect pathway is added to the SSs of P cells below the VPFL and before the filters described in the preceding text (Fig. 2).

\section{Model of synaptic plasticity in the cerebellar cortex}

To quantitatively simulate the changes of synaptic weights according to the physiologically known types of synaptic plasticity summarized in Table 1 (LTD, LTP, and RP), we had to model the "temporal window" explicitly. Two detailed studies on this are available. Chen and Thompson (1995) recorded changes in field potentials after stimulating GCAs and CFs with a wide range of time differences between their activations (from -250 to $250 \mathrm{~ms}$ ). They reported that LTD was maximally induced when GCA inputs were excited $250 \mathrm{~ms}$ before CF activation. Karachot et al. (1994) estimated the LTD size by recording changes in extracellular voltage, while periodically stimulating GCAs and CFs with a fixed time delay (GCAs were always delayed in their description). They reported that LTD occurred almost uniformly when the GCA inputs followed the $\mathrm{CF}$ input within a 2-s interval.

Therefore the conclusions of these two reports are in sharp contrast to one another. For our simulation, first, we use the Gaussian temporal window, which has its center $200 \mathrm{~ms}$ before the CF input and has a SD of $50 \mathrm{~ms}$. This is similar to the least-square fitting of a Gaussian function to the data of Chen and Thompson (1995), which results in a center at $211 \mathrm{~ms}$ before the CF input and an SD of $53 \mathrm{~ms}$. At the end of the simulation, we also attempt to use the temporal window, which is based on the interpretation of Karachot et al. (1994). It is spread uniformly between 0 and 2,000 ms after the CF input. The areas below the two temporal windows are both 1 . This means that the LTD change induced by one CS firing is the same between the two temporal windows. There have been no reports on temporal windows for LTP and RP. Accordingly, we use the LTD temporal windows for LTP and RP in our simulations.

TABLE 1. Reported changes in the synaptic transmission efficacy in the cerebellar cortex

\begin{tabular}{lll}
\hline \hline & \multicolumn{2}{c}{ Climbing Fiber } \\
\cline { 2 - 3 } & Fire & \multicolumn{1}{c}{ Silent } \\
\hline Parallel fiber fires & LTD & \multicolumn{1}{c}{ LTP } \\
Inhibitory cell fires & RP & No change \\
\hline
\end{tabular}

Reported changes in synaptic transmission efficacy in the cerebellar cortex. LTD, long-term depression (Ito and Kano 1982; Ito et al. 1982); LTP, longterm potentiation (Hirano 1990; Sakurai 1987); RP, rebound potentiation (Kano 1996; Kano et al. 1992). 
The following equation represents the change in synaptic weight of GCA inputs in LTD and LTP during each stimulus presentation:

$$
\begin{aligned}
& \frac{\mathrm{d} \omega_{\mathrm{GCA}}(t)}{\mathrm{d} t}=- 1 / n_{\mathrm{LTD}} \mathrm{GCA}(t) \int_{0}^{350} G\left(t-t^{\prime}\right) \cdot \mathbf{1}\left(\mathrm{CF}\left(t^{\prime}\right)-\mathrm{CF}_{\mathrm{sp}}\right) \mathrm{d} t^{\prime} \\
&+1 / n_{\mathrm{LTP}} \mathrm{GCA}(t) \int_{0}^{350} G\left(t-t^{\prime}\right) \cdot \mathbf{1}\left(\mathrm{CF}_{\mathrm{sp}}-\mathrm{CF}\left(t^{\prime}\right)\right) \mathrm{d} t^{\prime} \\
&-1 / \tau\left(\omega_{\mathrm{GCA}}(t)-\omega_{0}\right)
\end{aligned}
$$

Here

$$
\mathbf{1}(x)= \begin{cases}0, & \text { for } x<0 \\ x, & \text { for } x \geq 0\end{cases}
$$

In $E q .2, \omega_{\mathrm{GCA}}(t)$ denotes the synaptic weight between GCAs and P cells at time $t$. Here, the time origin is set at the stimulus motion onset for each trial. The first term is for the change caused by $\operatorname{LTD}$. $\mathrm{GCA}(t)$ and $\mathrm{CF}(t)$ denote the firing frequency at time $t$ for the GCA inputs and the CF inputs, respectively. CFsp, $1 / n_{\mathrm{LTD}}$, and $G(t)$ are the spontaneous firing frequencies of the CF inputs, the LTD coefficient, and the temporal window, respectively. The intuitive interpretation of the temporal window is easily understood. Each CF input spike occurring at time $t^{\prime}$ opens a temporal window described by $G\left(t-t^{\prime}\right)$, within which, if a GCA spike falls at time $t$, LTD is induced and its magnitude is proportional to the amplitude of $G\left(t-t^{\prime}\right)$ at that time difference $t-t^{\prime}$. LTD decreases $\omega_{\mathrm{GCA}}(t)$, in proportion to the product of the $\mathrm{CF}$ increase from its spontaneous level and GCA inputs. The second term represents the change caused by LTP. $1 / n_{\text {LTP }}$ denotes the coefficient for change caused by LTP. LTP increases $\omega_{\mathrm{GCA}}(t)$, in proportion to the product of the CF decrease from its spontaneous level and GCA inputs. The third term represents the exponential self-decay effect to the initial weight $\omega_{0}$. We estimate the time constant $\tau$ to be $\left(4.67 \times 10^{4}\right)$ [s] based on an experimental report stating that the experimentally induced OFR gain change diminishes by $1 / 3$ in one night (Miles and Kawano 1986).

The following equation represents the change in the synaptic weight of IC inputs in RP

$$
\begin{aligned}
\frac{\mathrm{d} \omega_{\mathrm{IC}}(t)}{\mathrm{d} t}=-1 / n_{\mathrm{RP}} \mathrm{IC}(t) \int_{0}^{350} G\left(t-t^{\prime}\right) \cdot \mathbf{1}\left(\mathrm{CF}\left(t^{\prime}\right)-\mathrm{CF}_{\mathrm{sp}}\right) \mathrm{d} t^{\prime} & \\
& -1 / \tau\left(\omega_{\mathrm{IC}}(t)-\omega_{0}\right)
\end{aligned}
$$

In $E q .3, \omega_{\text {IC }}(t)$ denotes the synaptic weight between IC and $\mathrm{P}$ cells at time $t$, which is negative. The first term is due to RP. $\operatorname{IC}(t)$ denotes the firing frequency at time $t$ for IC. $1 / n_{\mathrm{RP}}$ is the RP coefficient. RP increases the absolute value of $\omega_{\text {IC }}(t)$ (and decreases the value). The second term represents the self decay. Equation 3 does not contain a term corresponding to the second term in $E q .2$ because synaptic weights are not affected by IC stimulation in the absence of CFs (Table 1) (Kano 1996).

\section{METHODS}

The initial synaptic weights of 1,080 GCA and 1,080 IC inputs into 40 P cells, i.e., $\omega_{0}$ in Eqs. 2 and 3, were prepared as random numbers in the "inborn state," which simulates the P cells of an inborn monkey. The synaptic weight of each excitatory synapse was randomly chosen from a uniform distribution between 0.02 and 0.04. Similarly, the synaptic weight of each inhibitory synapse was randomly chosen from a uniform distribution between -0.02 and -0.04 . With these initial synaptic weights, only a slight SS firing modulation was produced by visual motion because the summation of the excitatory inputs and inhibitory inputs roughly canceled each other out.

The acquisition of normal OFR behaviors and SS firings was simulated by repetitively presenting simple ramp stimuli of various directions and speeds. Adaptations of OFR were simulated by repetitive presentations of velocity- and direction-step stimuli. Data recorded in a physiological experiment (Kobayashi et al. 1998) were used as the ramp stimuli at $10,20,40$, and $80 \%$ s. The ramp stimuli at other velocities, and the speed-step and direction-step stimuli, were synthesized from the preceding experimental data. A visual stimulus was fed into the model every $1 \mathrm{~ms}$, and the model generated MST, SS, and CS firing frequencies and eye movements every $1 \mathrm{~ms}$. The visual stimulus was given for $300 \mathrm{~ms}$, and the simulation was run for $350 \mathrm{~ms}$ because the latency from the visual stimulus to the eye movement was about 50 ms (Miles and Kawano 1986). After simulating the neural firing frequency for one trial with fixed synaptic weights, changes in synaptic weights were computed by Eqs. 2 and 3, and the new values were used for the next trial. There are no experimental reports on $n_{\text {LTD,LTP,RP }}$ in Eqs. 2 and 3. Thus we used $n$ that enables the simulated gain to near " 1 " after repeating the ramp stimuli: $\left(2.33 \times 10^{11}\right)$ for LTP, $\left(1.04 \times 10^{12}\right)$ for LTD, and $\left(1.04 \times 10^{12}\right)$ for RP. As a test stimulus to examine changes in firing and eye movements, we used a ramp stimulus for $150 \mathrm{~ms}$ at $10 \%$, called the test ramp stimulus. Matlab (MathWorks) was used on a Sun workstation.

In behavioral experiments on adaptation, 21,000 OFRs in four directions were elicited over 3 days while eliciting OFR gain changes (Miles and Kawano 1986). To simulate OFR acquisition by visual experiences after birth, a comparable number of repetitive visual stimuli presentations for five days were used, specifically, 36,000 ramp trials in four directions. A total of 900 trials of a $300-\mathrm{ms}$ ramp stimulus were repeated at 10 different velocities, i.e., every $10 \%$ from 10 to $100 \%$ s. The four stimulus directions were down, left, up, and right. The 900 trials $\times$ the 10 velocities $\times$ the four directions equals 36,000 trials.

\section{R E S U L T S}

\section{Acquisition of the ability to control OFR after birth}

After the 36,000 ramp trials, eye movements elicited by test stimuli in any direction increased stably to normal gain OFR in that direction because the synaptic weights asymptotically approached stable equilibrium values. For example, the maximum downward eye velocity elicited by the downward test ramp as a function of the learning trial count is shown in Fig. $5 A$. The mean modulation in the CF frequency of $20 \mathrm{v}-\mathrm{P}$ cells over the $350 \mathrm{~ms}$ after the onset of the upward stimulus motion of $10 \%$ s decreased to about one-half the initial level as the trial count increased (Fig. 5B). Therefore the "error-signal" encoded by $\mathrm{CF}$ modulation was diminished by learning. However, the $\mathrm{CF}$ modulation remained even after sufficient learning because there is no way to suppress the initial retinal error that appears before the eye movements. Figure $5, C-F$, shows the average $\mathrm{SS}$ temporal waveforms of 10 left v-P cells $(C$ and $E$ ) and the corresponding eye movements $(D$ and $F$ ) during the downward test stimulus, before $(C$ and $D)$ and after learning ( $E$ and $F$ ). Before repeating the ramp trials (at birth), the SS firing frequency of none of the $\mathrm{V}-\mathrm{P}$ cells is well modulated during any of the visual stimuli (Fig. $5 C$ ); therefore the eyes did not move much (Fig. 5D). After repeating the ramp trials, the SS firing frequencies of the v-P cells were well modulated (Fig. $5 E$ ); the eyes followed the visual motion quite well (Fig. $5 F$ ). Figure $5 G$ 

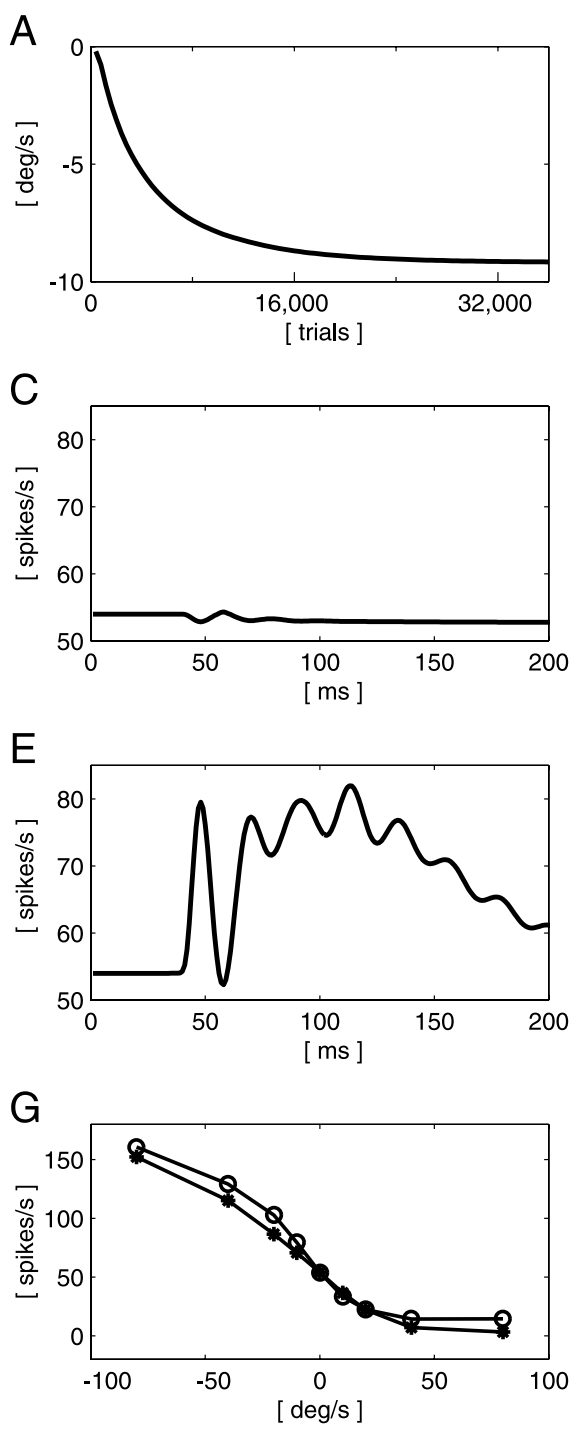

plots the SS frequency averaged over the time interval between 50 and $150 \mathrm{~ms}$ of stimulus motion onset as a function of the vertical stimulus velocity. The figure compares experimental data from adult monkeys (Kobayashi et al. 1998) with the average of simulated results from $10 \mathrm{P}$ cells at the end of OFR acquisition. The simulated results denoted by "*" reproduced the experimental data $(\bigcirc)$ well. The preferred velocity of SSs became the high velocity of $80 \%$ s for all of the $\mathrm{P}$ cells, in agreement with the experimental data (Shidara and Kawano 1993).

Figure $6 A$ shows the acquired temporal firing frequency patterns of SSs and CSs of 10 left v-P cells in response to the upward test ramp. Although the amplitudes of the SS modulation varied greatly, the temporal waveforms of SSs and CSs were very stereotypical. Furthermore, P cells with large CS modulations exhibited large SS modulations. Similarly, P cells with smaller CS modulations exhibited smaller SS modulations. There was a statistically significant cell-to-cell negative correlation $(-0.92, P=0.001)$ between the maximum modulations of SSs and the modulations in the mean firing frequency of CSs over a period of 50-150 ms after stimulus onset (Fig. $6 B)$. This statistically significant negative correlation of indi- vidual cell characteristics between SS and CS modulations has also been found in monkeys (Kobayashi et al. 1998).

Figure 7 shows the directional selectivity of the four groups of $P$ cells before and after repeating the 36,000 ramp stimuli. In Fig. 7, the blue and red circles connect points that show the maximum and minimum SS firing rates in response to the test ramp in a given direction on a polar plot, before and after learning, respectively. The red arrows are vectorial sums of all of the red circle points and show the preferred directions for the stimulus motion. Before learning, the SSs were not modulated to any stimulus direction (blue circles); the population vector was almost zero. Therefore blue arrows are not seen. After learning, however, the mean preferred directions of the left and right $\mathrm{v}-\mathrm{P}$ cells became downward and were $266.87 \pm 6.17^{\circ}$ and $260.85 \pm 14.61^{\circ}$ (mean $\left.\pm \mathrm{SD}\right)$, respectively (Fig. 7, $A$ and $B$ ) when $0^{\circ}$ is defined as rightward and the angle is measured in a counterclockwise rotation. The mean preferred direction of the left h-P cells became leftward at $176.98 \pm 12.94^{\circ}$ (Fig. 7C). The preferred direction of the right h-P cells became rightward at $353.78 \pm 8.43^{\circ}$ (Fig. 7D). As can be seen from the red circles, the directional tuning curves of all of the $\mathrm{P}$ cells after OFR acquisition were well-fitted by full cosine functions in 
A
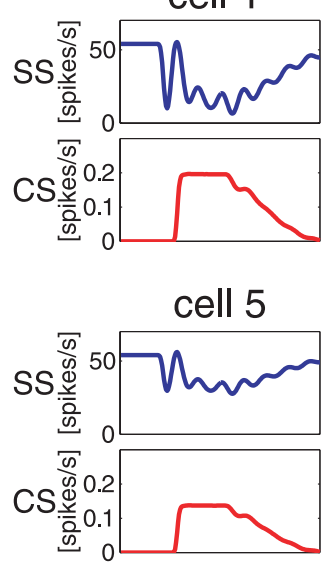

cell 9

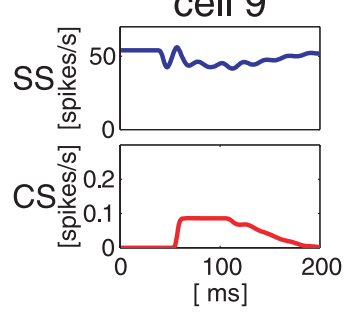

cell 2

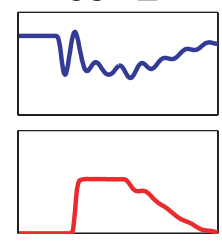

cell 6

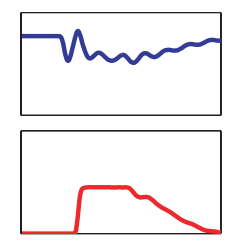

cell 10

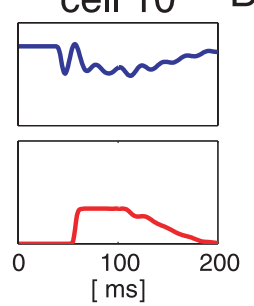

cell 3

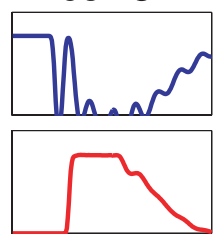

cell 7

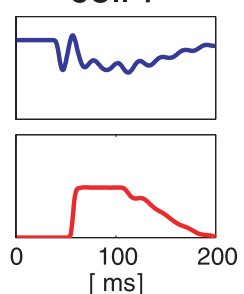

B

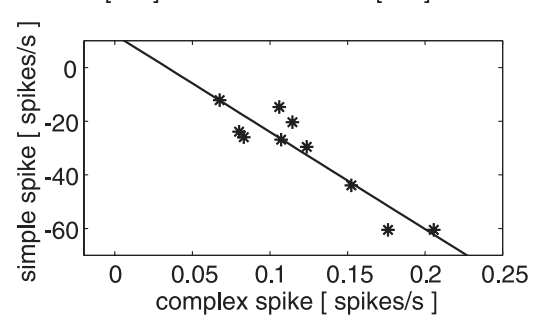

cell 4

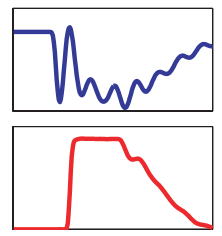

cell 8
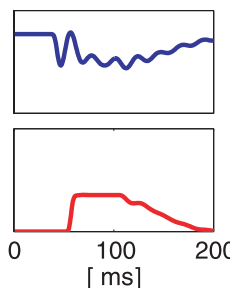

FIG. 6. Reciprocal relationships between simulated SS and CS firing frequencies as a result of the simulation. A: temporal firing frequency patterns of the SSs (top, blue curves) and CSs (bottom, red curves) of $10 \mathrm{v}-\mathrm{P}$ cell models on the left side with an upward $10 \%$ ramp stimulus after repeated ramp trials. The patterns of CSs are indicated as increases from their spontaneous firing frequencies. $B$ : maximum modulation (decrease) of the SS firing frequencies plotted against the average modulation (increase) of CSs during the 50-150 ms after stimulus onset for each of the $10 \mathrm{P}$ cells during the upward $10 \%$ ramp stimulus. The solid line represents the linear regression of the data $(n=10 ; r=-0.92)$. agreement with the experimental data of Shidara and Kawano (1993). The acquired preferred direction of SSs was opposite to that of CSs for each P cell. This agreed well with the monkey data (Kobayashi et al. 1998).

Figure 8, $A$ and $B$, represents the changes in synaptic weights from GCAs and ICs. The average weights of the synapses are graphically represented as the diameters of the open small
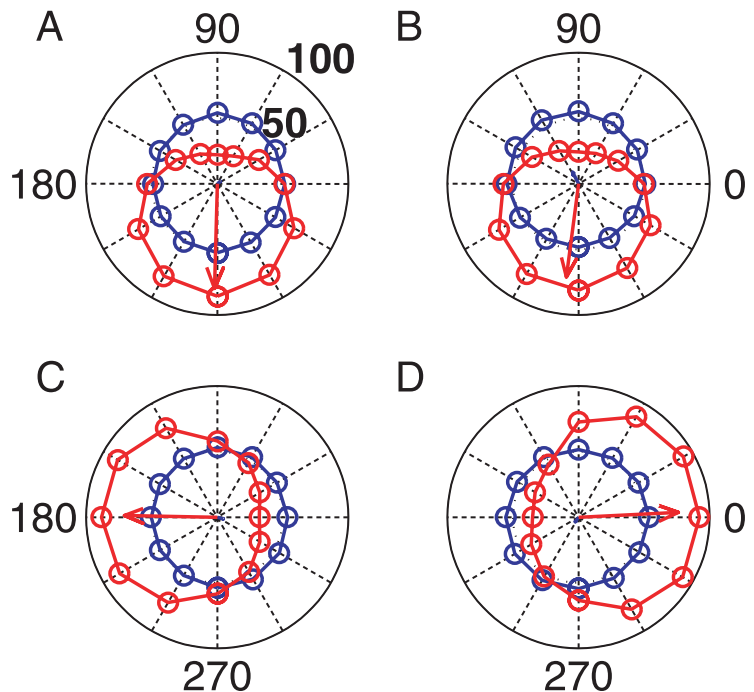

FIG. 7. Direction-dependent SS activation and preferred directions of the $\mathrm{P}$ cells before (blue circles) and after (red circles and arrows) repeated ramp trials. $A-D$ : the averages of 10 left v-P cells, 10 right v-P cells, 10 left h-P cells, and 10 right h-P cells, respectively. The circles denote the SS frequency averaged over the time interval between 50 and $150 \mathrm{~ms}$ after the stimulus motion onset for stimuli in 12 different directions. When the maximum modulation was 100 spikes/s, a plotted point appears on the outer filled circle. The red arrows denote the average preferred directions for each of the 4 groups of $P$ cells. They are defined as 2 -dimensional vectorial summations of 12 points and are enlarged by a scale of 5 for clear viewing. circles (excitatory) and filled small circles (inhibitory). The relative position of each small circle with respect to the location of the $\mathrm{P}$ cell (shown as an open large circle) represents the preferred direction for that synaptic input. Before repeating the stimuli (Fig. 8A), the average weights of the excitatory and inhibitory synapses were very similar among all of the preferred directions. Although the initial synaptic weight for each individual synapse was chosen randomly, the displayed averages were taken from 900 synapses (10 P cells, 30 preferred velocities, 3 waveforms), and gave similar values. Due to these spatially uniform weights, no $\mathrm{P}$ cell exhibited strong SS modulation to any stimulus before learning (Fig. 5C). After learning (Fig. 8B), the excitatory and inhibitory synapses changed their weights. LTP increased the excitatory synaptic weights (graphically, enlarging the open circles) and induced SS enhancement with the stimulus in the preferred direction of the GCA inputs. LTD decreased the excitatory synaptic weights (graphically, shrinking the open circles) and induced SS suppression with the stimulus in the no-preferred direction of GCA. RP increased the inhibitory synaptic weights (graphically, enlarging the filled circles) and induced SS suppression with the stimulus in the nonpreferred direction of IC inputs. Overall, the synaptic weight changes shown in Fig. $8 B$ built up the downward preferred direction for $\mathrm{v}-\mathrm{P}$ cells, the leftward preferred direction for left h-P cells, and the rightward preferred direction for right h-P cells, which are shown in Fig. 7.

Figure $8 C$ schematically illustrates how the modifications of the excitatory and inhibitory synaptic weights shown in Fig. 8B were induced by the interaction between the GCA and IC inputs and the $\mathrm{CF}$ input for a v-P cell. The temporal firing patterns of the GCA and IC inputs with preferred downward and upward directions are shown in the first and second rows, respectively, in Fig. $8 C$. The third row shows the temporal pattern of the CF input with an upward preferred direction for 
A

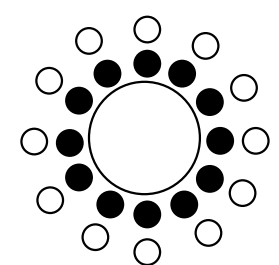

B

a
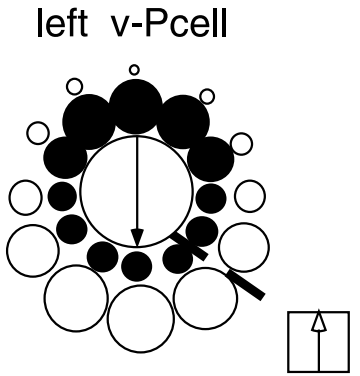

I.O.

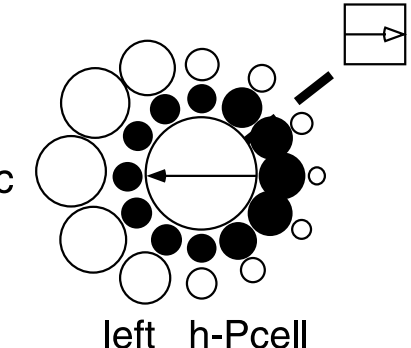

b

right v-Pcell
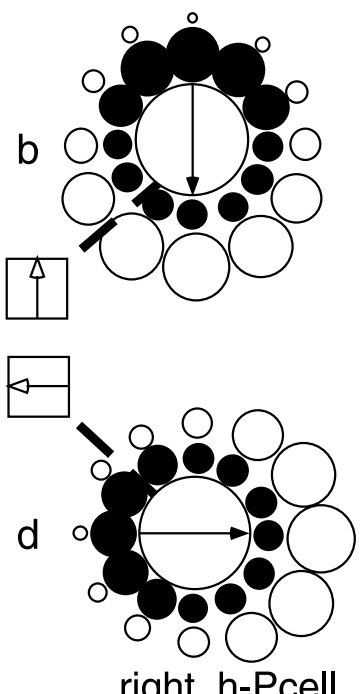

right $\mathrm{h}$-Pcell

C

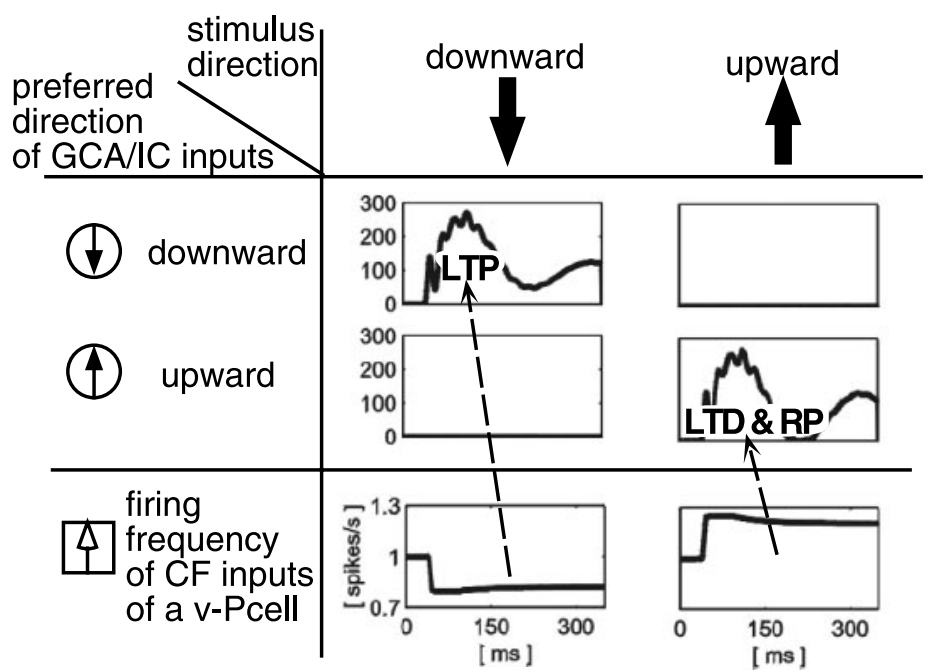

FIG. 8. Visualized presentation of synaptic weights on P cells before $(A)$ and after $(B)$ learning and the synaptic plasticity mechanisms involved in this change $(C)$. The large circles denote $\mathrm{P}$ cells. The diameters of the open and filled small circles denote the synaptic weights with the transformation described below. For clarity, the diameters of the circles were determined from the corresponding synaptic weights $\omega$ as follows: diameter $=(\omega-$ $\left.\omega_{0}\right) \times 100+\omega_{0}$. Here, $\omega_{0}$ was the initial synaptic weight. The open, small circles are average weights of granule cell axons (GCA) excitatory synapses, and the filled, small circles are average weights of inhibitory inhibitory cell (IC) synapses. The positions of the small circles with respect to the open, large circles indicate the preferred directions of these synaptic inputs. The arrows within the large circles (P cells) show the acquired mean preferred directions of SSs. A: before repeated ramp trials, the average synaptic weights were similar in all directions for all $\mathrm{P}$ cells. $B$ : after repeated ramp trials, $a-d$ : the results for the left $\mathrm{v}-\mathrm{P}$ cell, right $\mathrm{v}-\mathrm{P}$ cell, left h-P cell, and right h-P cell, respectively. IO, inferior olive, and the 4 arrows within the squares at the center of $B$ denote the preferred directions of the $\mathrm{CF}$ inputs. $C$ : simulated GCA, IC, and CF firing frequency patterns and synaptic plasticity mechanisms in the "inborn state" model during downward and upward stimuli. The 1st and 2nd rows show temporal patterns of GCAs and ICs with downward and upward preferred directions, respectively. While 2,160 GCAs and ICs exist in the model, only the 2 with preferred velocities of $10 \%$, with a small phasic firing and with an upward or downward preferred direction, are shown. The 3rd row shows the temporal patterns of CF inputs for v-P cells. Left and right: temporal firing frequency patterns with downward and upward stimuli, respectively. The 2 broken-line arrows indicate that the designated synaptic plasticity is induced by the interaction between the GCA/IC inputs and CF inputs. $D$ : changes in synaptic weight for each preferred velocity. The ordinate denotes the average change of synaptic weights from the initial weight. The abscissa denotes preferred velocity of the GCA/IC inputs. The averaging was computed for 2,880 synapses (40 P cells, excitatory and inhibitory, 12 directions, 3 firing patterns) for each preferred velocity.

D

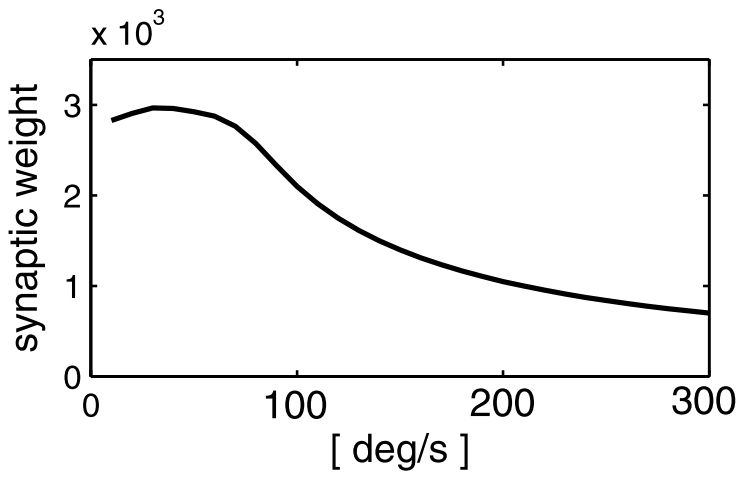


the v-P cell. The left and right columns show the temporal firing patterns when the stimulus moved downward and upward, respectively. When the stimulus was downward (left), the $\mathrm{CF}$ firing frequency decreased below the spontaneous firing frequency (3rd row, left). Then, the conditions for the induction of LTP (summarized in Table 1) were achieved for the GCA synapses with a downward preferred direction as shown by the broken-line arrow in the left column. Because we used the temporal window that occurs mainly before $\mathrm{CF}$ modulation, the broken-line arrow goes from the future in $\mathrm{CF}$ time to the past in GCA input time, indicating that the GCA inputs preceding the CF inputs underwent LTP. When the stimulus was upward (right), the $\mathrm{CF}$ firing frequency increased (3rd row, right). Then, the conditions for the induction of LTD were satisfied for the GCA synapses with an upward preferred direction. Furthermore, the conditions for the induction of RP were satisfied for the IC synapses with an upward preferred direction. The induction of LTD and RP is schematically shown by the broken-line arrow in the second column. When the stimulus was leftward or rightward, the firing frequency of CFs of the v-P cell was not modulated. As a result, no change in synaptic weight was induced for either the GCA or IC inputs. The synaptic weights of the GCA and IC inputs with leftward or rightward preferred directions were not modified because the CF input was not statistically modulated on average when the GCA and IC inputs were modulated. This is because the 36,000 stimulus motions were statistically uniform in their directional distributions.

Very similar combinations of LTP, LTD, and RP resulted in the synaptic weight distributions shown in Fig. $8 B c$ for left h-P cells and right h-P cells. For h-P cells, the $\mathrm{CF}$ input was modulated only by a horizontal component of visual motion. The CF firing increased with contralateral stimuli and decreased with ipsilateral stimuli. Therefore LTP was induced by synapses from GCAs with preferred ipsilateral directions. LTD and RP were induced by synapses from GCAs and ICs with preferred contralateral directions. Synapses from GCAs and ICs with preferred upward or downward directions were not modified because the $\mathrm{CF}$ inputs to the h-P cells were not modulated by vertical visual motion.

Figure $8 D$ shows the average weight of synapses at each preferred velocity. The average change in synaptic weight from the initial values was taken from 1,440 synapses (40 P cells, 12 preferred directions, 3 waveforms). Figure $8 D$ reveals a relationship like an exponential decrease in synaptic weight for the inputs at each preferred velocity. The weights for low preferred velocities were high, and those for high velocities were low. These exponentially learned synaptic weights might be the origin of the high preferred velocity of SSs and the saturation of SSs that is simulated in Fig. 5G. The velocity of retinal error was almost the same as the visual stimulus velocity $50 \mathrm{~ms}$ after stimulus onset. Meanwhile, the retinal error velocity decreased 50-150 ms after stimulus onset because of the eye movement. This might mean that firing occurs not only in inputs with preferred velocities the same as the stimulus velocity but also in inputs with preferred velocities smaller than the stimulus velocity. The integration of the exponential decrease from 0 to the stimulus velocity increases logarithmically. Thus the firings might show a logarithmic increase when the synaptic weights show an exponential spread at the preferred velocity. Such logarithmic increases might cause the high preferred velocity of SSs and the saturation of SSs.
After OFR acquisition, simulated SS firing was reconstructed by the inverse dynamics model of simulated eye movement, that is, by a linear combination of acceleration, velocity, and position. This analysis followed previous monkey studies (Gomi et al. 1998; Shidara et al. 1993). The estimated coefficients of acceleration, velocity, and position were $0.112 \pm 0.074($ mean \pm SD), $2.61 \pm 1.442$, and $-6.97 \pm 4.27$, respectively. The squared correlation coefficient between the simulated firing and the reconstructed firing was $0.993 \pm$ 0.006 . The ratio of the acceleration/velocity coefficients was $0.0441 \pm 0.0125$. This was in good agreement with the ratio (0.0502) obtained from the experimental data that we used to construct the model (Yamamoto et al. 1997). Consequently, our model succeeded in simulating the acquisition of OFR, given the temporal window of plasticity that spread mainly before $\mathrm{CF}$ was used. From this point on, the set of synaptic weights obtained after 36,000 ramp trial repetitions will be called the "adult state," because our model with this set captured well the OFR characteristics and P-cell firings of adult monkeys.

\section{Adaptation of OFR gain}

After the simulation of OFR acquisition, the step-up stimuli (upward and rightward) and the step-down stimuli (downward and leftward) were presented to the model, following the protocol of a monkey experiment (Miles and Kawano 1986). For a detailed explanation of the speed-step stimuli, see Ocular following responses: behavioral and neurophysiological studies. A total of 250 trials were repeated for each of seven velocities $10,20,30,40,60,80$, and $100 \%$ s, in one of four directions. Altogether, 7,000 trials were repeated, just as in the monkey adaptation experiment in one day (Miles and Kawano 1986). We used the final synaptic weights $\omega_{\mathrm{GCA}}$ and $\omega_{\mathrm{IC}}$ obtained in the OFR acquisition simulation, that is, the adult state as the initial values of synaptic weights $\omega_{0}$ in Eqs. 2 and 3.

Figure 9, $A$ and $B$, shows the behaviors of the model system during the speed-step adaptation simulation to the downward and upward test ramps, respectively. The gain to the downward test ramp decreases from 0.92 to 0.54 (Fig. 9A $a$ ), while the gain to the upward test ramp increases from 0.99 to 1.77 (Fig. 9Ba). In response to the downward test ramp (Fig. 9A), the SSs decrease their firing frequency (from $b$ to $c$ ), and the downward eye movement becomes smaller (from $d$ to $e$ ). On the other hand, in response to the upward test ramp (Fig. 9B), the SSs increase their firing suppression (from $b$ to $c$ ) and the upward eye movement becomes larger (from $d$ to $e$ ).

Figure $9 C$ shows the simulated GCA, IC, and CF inputs to a v-P cell of the adult state model at the beginning of speedstep adaptation and schematically illustrates how modifications of the excitatory and inhibitory synaptic weights were induced by the interaction between these inputs. The format of Fig. 9C is similar to that of Fig. $8 C$. The left and right columns correspond to the downward step-down stimulus and upward step-up stimulus, respectively. For the downward step-down stimulus, the CS firing frequency decreased below its spontaneous level for the first $150 \mathrm{~ms}$. However, it increased over its spontaneous level for the next $150 \mathrm{~ms}$ because the downward eye velocity surpassed the reduced stimulus velocity and generated the upward retinal error (3rd row, left). GCAs and ICs 
A
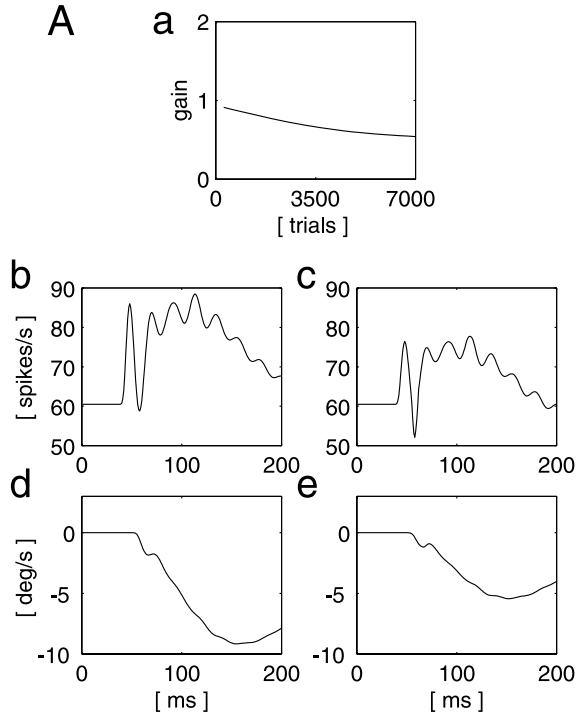

e

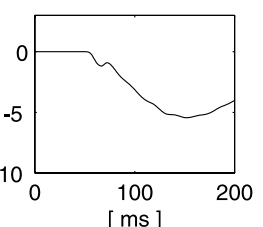

B
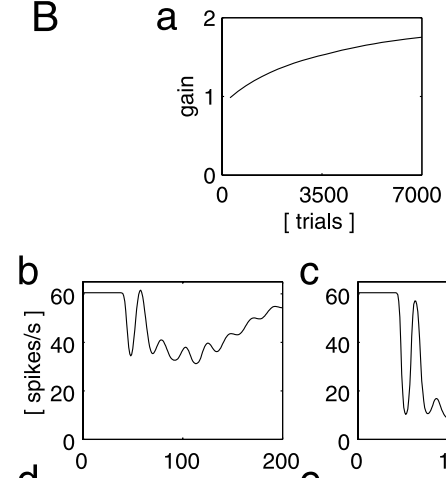

c

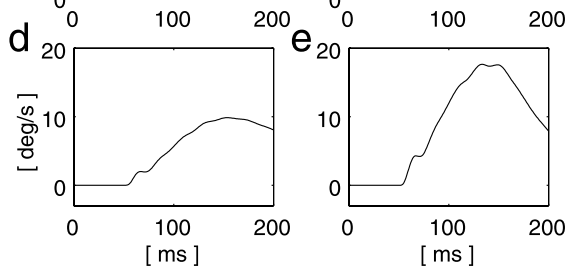

C

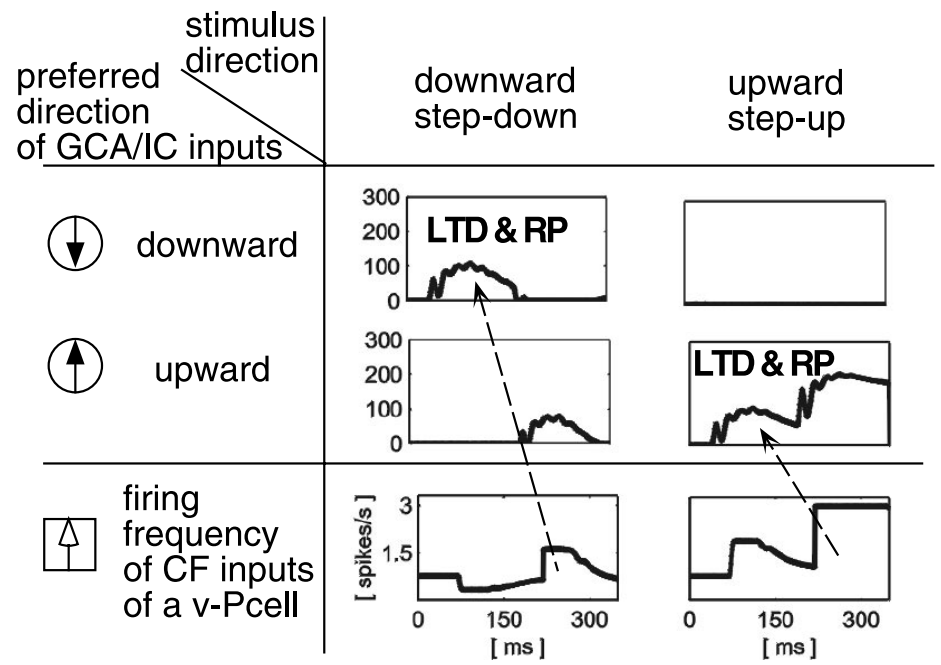

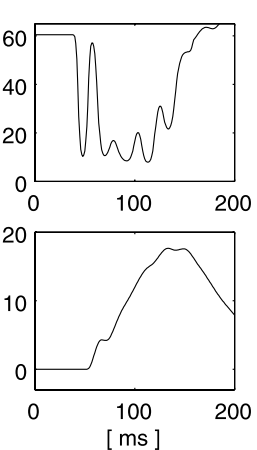

FIG. 9. Simulation results for gain adaptation. A: changes observed for a $10 \%$ downward test ramp. $B$ : changes observed for a $10 \%$ s upward test ramp. $a$ : changes in the OFR gain as a function of the speed-step trial number. $b$ and $c$ : average SS firing frequencies of $20 \mathrm{v}-\mathrm{P}$ cells before $(b)$ and after $(c)$ repeated speed-step trials. $d$ and $e$ : temporal patterns of eye velocities before $(d)$ and after $(e)$ repeated speed-step trials. A positive eye velocity indicates upward motion. From $b$ to $e$, the abscissa denotes the time after the stimulus motion onset in ms. $C$ : simulated GCA, IC, and CF firing frequency patterns and synaptic plasticity mechanisms in the "adult state" model during $40 \%$ s speed-step stimuli. The format of this figure is similar to that of Fig. $8 C$. The 1 st and 2 nd rows show temporal patterns of GCAs and ICs with preferred downward and rightward directions, respectively, and a preferred velocity of $100 \%$ s. Left and right: temporal firing frequency patterns with downward step-down and upward step-up stimuli, respectively. with downward preferred directions increased their firing frequencies for the first $150 \mathrm{~ms}$ (1st row, left). As summarized in Table 1, LTD and RP were induced at the synapses from GCAs and ICs that had a downward preferred direction and fired in the first $150 \mathrm{~ms}$. The broken-line arrow indicates that LTD and $\mathrm{RP}$ were induced between the preceding GCA and IC activities with a subsequent increase in CF input according to the temporal window that occurs before CF modulation. The LTD and RP that occurred on synapses with a downward preferred direction reduced the SS firing frequency in $\mathrm{v}-\mathrm{P}$ cells for the downward stimulus and decreased the eye movement gain (Fig. 9A). Here, we must note that any synaptic plasticity induced by the $\mathrm{CF}$ activity occurring in the first $150 \mathrm{~ms}$ need not be considered here for adaptation because its influence has already been incorporated in the equilibrium adult state of synaptic weights. In other words, its effects have already been balanced with the other terms in Eqs. 2 and 3 at the beginning of the adaptation simulation.

For the upward step-up stimulus, the CSs increased their firing frequency above the spontaneous level for $300 \mathrm{~ms}$, especially in the last $150 \mathrm{~ms}$ (Fig. $9 C, 3 \mathrm{rd}$ row, right). This is because the upward stimulus velocity was increased, and a larger retinal error was generated. The GCAs and ICs with upward preferred directions increased their firing frequencies over the entire $300 \mathrm{~ms}$ (2nd row, right). As summarized in Table 1, LTD and RP were induced at synapses from these GCAs and ICs (broken-line arrow) because of the temporal window that occurs mainly before CF modulation. These instances of LTD and RP increased the magnitude of the SS suppression induced by the upward test ramp in the v-P cells and increased the eye movement gain (Fig. 9B).

In essentially the same manner, the gain to the leftward test ramp decreased from 0.89 to 0.69 , and the gain to the rightward test ramp increased from 0.95 to 1.65 (data not shown). When the stimulus was a leftward step-down stimulus, LTD and RP were induced in left h-P cells on synapses from GCAs and ICs with preferred leftward directions. These instances of LTD and RP decreased the SS firing frequency in the left h-P cells and decreased the OFR gain to the leftward stimulus. When the stimulus was the rightward step-up stimulus, LTP was induced in right h-P cells on synapses from GCAs with a preferred rightward direction. This LTP increased the SS firing frequency in the right h-P cells and increased the OFR gain to the rightward stimulus. Consequently, our model with adult-state initial synaptic weights reproduced the main characteristics of the gain adaptation experiment by Miles and Kawano (1986) 
when the temporal window that occurs mainly before $\mathrm{CF}$ modulation was used.

\section{Adaptation of OFR to directional step stimuli}

Independently of the speed step simulations, after the simulation of OFR acquisition, adaptation to directional steps was simulated following the protocol of a monkey experiment (Miles and Kawano 1986). Down-right, left-down, up-left, and right-up direction step stimuli were presented to the adult state model at $10,20,30,40,60,80$, and $100 \%$ s. In the down-right trial, a ramp stimulus was downward for the first $150 \mathrm{~ms}$, and then the direction was changed to rightward for the next 150 ms. In the left-down, up-left, and right-up trials, a left, up, or right ramp stimulus was given for the first $150 \mathrm{~ms}$ and then a down, left, or up ramp stimulus was given for the next $150 \mathrm{~ms}$, respectively. A total of 250 trials was repeated for the direction steps of each type. Altogether, there were 7,000 trials (7 speeds, 4 directions, 250 trials). As the initial values of synaptic weights $\omega_{0}$ in Eqs. 2 and 3, we used the final synaptic weights $\omega_{\mathrm{GCA}}$ and $\omega_{\mathrm{IC}}$ obtained in the OFR acquisition simulation, that is, the adult state.

After repeated counterclockwise directional step stimuli, the directions of the eye movements for the test ramp were changed to counterclockwise. The eyes moved down-rightward $\left(305.8^{\circ}\right)$ for the downward stimulus, left-downward $\left(225.1^{\circ}\right)$ for the leftward stimulus, up-leftward $\left(130.6^{\circ}\right)$ for the upward stimulus, and right-upward $\left(35.8^{\circ}\right)$ for the rightward stimulus. Figure 10A, $a$ and $b$, shows the horizontal (solid curve) and vertical (broken curve) eye velocities during the leftward test ramp stimulus of $10^{\circ} / \mathrm{s}$, before $(a)$ and after $(b)$ the directional step adaptation. Before the adaptation, the leftward test stimulus evoked little vertical movement (broken curve in Fig. $10 \mathrm{Aa}$ ). On the other hand, a clear downward component emerged after the adaptation (broken curve in Fig. 10Ab). Figure $10 \mathrm{Ac}$ shows the two-dimensional plot of eye velocity after the adaptation.

The SS activities of left $10 \mathrm{v}$-P cells to the different directional stimuli and their mean preferred direction vector before the adaptation, shown as the red circles and arrow in Fig. 10B, changed after the adaptation to the results represented by the green circles and arrow. The preferred directions of the left and right v-P cells changed from downward to down-leftward $233.78 \pm 8.28^{\circ}$ (Fig. $10 B$ ) and $227.21 \pm 17.06^{\circ}$ (means \pm $\mathrm{SD})$. The v-P cells with down-leftward preferred direction increased their SS firing for even the leftward stimulus to which they were not responsive at all before the adaptation. This increase in the SS firings of the v-P cells then induced downward eye movements through the fixed brain stem network. Therefore the downward component of the eye movements was induced in response to the leftward stimulus (Fig. $10 \mathrm{Ab}$ ). The preferred direction of the left h-P cell changed from leftward to left-upward $139.99 \pm 9.90^{\circ}$. The preferred direc-
A

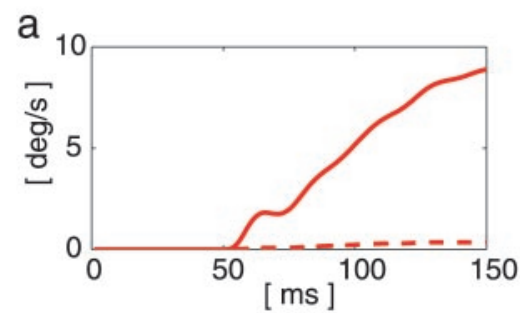

b

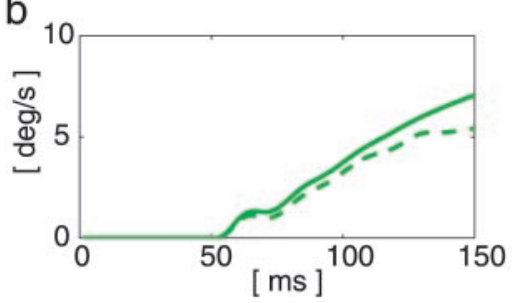

C

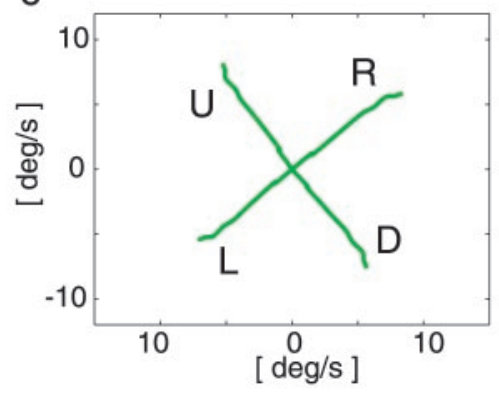

B

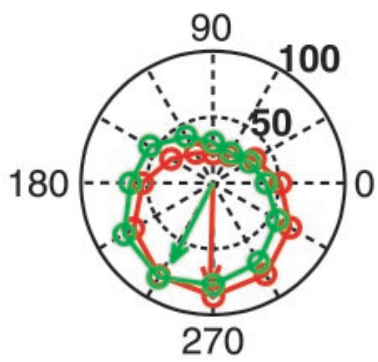

C

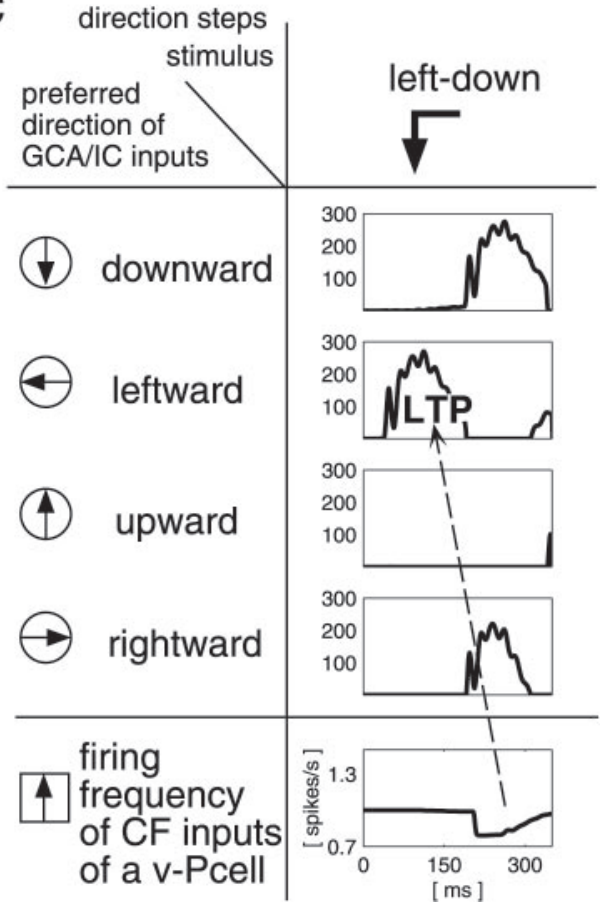

FIG. 10. A: temporal patterns of eye velocity in response to a leftward $10 \%$ ramp stimulus before (red curves in $a$ ) and after (green curves in $b$ ) repeated direction-step stimuli. The horizontal component of the eye velocity is shown by the solid curve, where positive values represent leftward motion. The vertical component of eye velocity is shown by the broken curve, where positive values represent downward motion. $c$ : a 2-dimensional plot of eye velocity [in the same way as the monkey's report, cf. Fig. 12, $B$ and $C$, of Miles and Kawano (1986)] showing the trajectories evoked by $10 \%$ ramp stimuli after repeated direction-step stimuli. The plots labeled R, L, $\mathrm{U}$, and D are for eye velocity for right, left, up, and down test ramp stimuli, respectively. Each plot starts at 0 velocity at the center. $B$ : simulated directional tuning curve and preferred direction of the average SS from 10 left $\mathrm{v}$-P cell models before (red circles and arrow) and after (green circles and arrow) repeated direction-step trials. The format is similar to that of Fig. 7. $C$ : simulated firing frequency patterns and synaptic plasticity induced in the adult state model for left-down direction-step stimuli. The format is similar to that in Fig. $8 C$ but is more comprehensive. The 1 st-4th rows show temporal patterns of GCA/IC inputs with downward, leftward, upward, and rightward preferred directions, respectively, as well as CF inputs for a v-P cell. The column shows temporal firing frequency patterns for left-down step. The broken-line arrow indicates that the designated synaptic plasticity is induced by the interaction between the GCA/IC inputs and CF inputs. 
tion of the right h-P cell changed from rightward to rightdownward $319.99 \pm 8.43^{\circ}$.

Figure $10 C$ shows the simulated GCA, IC, and CF inputs of a $\mathrm{v}$-P cell of the adult state model during left-downward step stimuli at the beginning of the directional step adaptation in a format similar to that of Figs. $8 C$ and $9 C$. However, Fig. $10 C$ is more complete, in that it includes four kinds of GCA/IC inputs. Due to the temporal window that occurs mainly before $\mathrm{CF}$ modulation, the CF modulation in the last $150 \mathrm{~ms}$ induced LTD, RP, or LTP on synapses from the GCA and IC inputs that were activated in the first $150 \mathrm{~ms}$ as shown by the broken-line arrow. When the stimulus was left-down steps, the CS decrease in the last $150 \mathrm{~ms}$ (5th row) induced LTP on synapses from GCAs with preferred leftward directions (2nd row). This LTP increased the SSs of v-P cells in response to the leftward stimulus, and the eyes started to move downward with the leftward stimulus. When the stimulus was right-up steps, the CS increase in the last $150 \mathrm{~ms}$ induced LTD and RP on synapses from GCAs and ICs with preferred rightward directions. This LTD and RP decreased the SSs of v-P cells in response to the rightward stimulus, and the eyes started to move upward with the rightward stimulus. Altogether, LTD, LTP, and RP induced changes in the preferred directions of v-P cells, as shown in Fig. 10B. LTD, LTP, and RP modified the preferred directions of h-P cells in a similar way.

Consequently, our model succeeded in reproducing the main behavioral data obtained in directional adaptation for visuomotor transformations in OFR (Miles and Kawano 1986) based solely on cerebellar plasticity using the temporal window that occurs mainly before CF modulation. Furthermore, our model nonintuitively predicts that the preferred direction of $\mathrm{P}$ cells changes to clockwise, and the direction tuning curves also rotate clockwise as a whole (Fig. 10B) when the counterclockwise directional adaptation of eye movements is induced by counterclockwise direction-step stimuli.

\section{Temporal window that allows only CF inputs preceding GCA and IC inputs}

To understand the role of the temporal window that allow only CF inputs preceding GCA and IC inputs, the three kinds of simulation experiments were repeated using another temporal window that occurs only after CF modulation based on the report by Karachot et al. (1994). $n$ in Eqs. 2 and 3 was set to be $\left(9.34 \times 10^{11}\right)$ for LTP $\left(n_{\mathrm{LTP}}\right)$, and $\left(5.02 \times 10^{12}\right)$ for LTD $\left(\mathrm{n}_{\mathrm{LTD}}\right)$ and $\mathrm{RP}\left(n_{\mathrm{PR}}\right)$.

First, the OFR acquisition was completed successfully in a manner similar to that used in the previous simulation. The mean preferred direction of the left and right v-P cells became downward at $270.22 \pm 5.42$ and $265.68 \pm 10.99^{\circ}$, respectively. The mean preferred direction of the left h-P cells became leftward at $180.89 \pm 9.86^{\circ}$. The preferred direction of the right h-P cells became rightward at $357.72 \pm 6.82^{\circ}$.

Second, the upward step-up adaptation reproduced was smaller than in Fig. $9 B$ or in the experimental data (Miles et al. 1986). The downward step-down adaptation was not reproduced. The gain to the upward test ramp increased from 1.04 to 1.31 , but the gain to the downward test ramp also increased from 1.05 to 1.18 .

Using a temporal window that only occurs after CF modulation, CF modulation can only influence the synapses of
GCAs and ICs that fire after CF modulation. When the stimulus was downward, the increase of the CF modulation in the last $150 \mathrm{~ms}$ (Fig. 9C, left, 3rd row) could not induce LTD or RP on synapses from GCAs and ICs with downward-preferred directions, which is why the gain decrease was not reproduced in this simulation. When the stimulus was upward, the increase of the CS modulation in the last $150 \mathrm{~ms}$ (Fig. 9C, right, 3rd row) induced LTD and RP from GCA/IC synapses with upward preferred directions. The LTD and RP generated the increase in the gain to the upward stimulus because the SS suppression was increased.

Third, the adaptation to the direction-step stimuli of Miles and Kawano (1986) was not reproduced. After repeated counterclockwise directional step stimuli, the direction of the eye movements to the test ramps changed to the clockwise direction, the opposite to the direction observed in the experimental data. The eyes moved down-leftward $\left(255.2^{\circ}\right)$ for the downward stimulus, left-upward $\left(162.7^{\circ}\right)$ for the leftward stimulus, up-rightward $\left(82.1^{\circ}\right)$ for the upward stimulus, and right-downward $\left(355.7^{\circ}\right)$ for the rightward stimulus.

The preferred directions of the $\mathrm{P}$ cells changed to counterclockwise, which was the opposite to that of the previous simulation results shown in Fig. 10B. Specifically, the preferred directions of the right and left v-P cells changed from downward to down-rightward at $281.89 \pm 4.18$ and $279.68 \pm$ $7.67^{\circ}$, respectively. The preferred direction of the left h-P cells changed from leftward to left-downward at $193.25 \pm 8.44^{\circ}$. The preferred direction of the right h-P cells changed from rightward to right-upward at $10.28 \pm 5.98^{\circ}$. Note that the changes in the preferred directions were small and opposite in rotation to those simulated by the temporal window that occurs mainly before $\mathrm{CF}$ modulation.

As opposed to the temporal window that occurs mainly before CF modulation, the temporal window that occurs after CF modulation allows the preceding CF modulation to influence the synaptic efficacy of GCA/IC inputs occurring afterward. Therefore when stimulus was down-right step stimulus, the decrease in CF firing on the v-P cells in the first $150 \mathrm{~ms}$ induces LTP on synapses from GCAs with rightward preferred directions that fire in the last $150 \mathrm{~ms}$. This results in the change of the preferred direction of the $\mathrm{v}-\mathrm{P}$ cells to a down-rightward direction, and the eyes tend to move downward in response to the rightward stimulus.

\section{I S C U S S I O N}

Based on previous behavioral, physiological, and computational studies on OFR, a large-scale network model for twodimensional OFR was developed and its adaptation was examined. The model included retinal error, the MST-DLPN, VPFL P cells, the accessory optic system, the CF system, and the oculomotor plant. At the beginning of the simulations, the $\mathrm{P}$ cell synaptic weights were small random values, and they were updated according to LTD, LTP, and RP using the temporal window that occurs mainly before CF modulation. With this realistic and quantitative model, OFR acquisition after birth was first successfully simulated. The adult state of the model after 36,000 presentations of ramp stimuli reproduced monkey behavioral data well, as well as the firing characteristics of MST and VPFL neurons during OFR. Specifically, we found that drastic transition in neural representation from the popu- 
lation codes in the MST to firing rate codes in VPFL regarding preferred directions, preferred velocities, and temporal waveforms of firings (Kawano 1999; Kawato 1999; Takemura et al. 2001) were learned at GCA and IC synapses on P cells. Approximately mirror-image relationships between SS and CS firing patterns on an individual cell basis (Kobayashi et al. 1998) were also reproduced. Second, the adaptation of OFR gains induced by speed steps was successfully reproduced. Third, the adaptation of OFR kinematic characteristics induced by direction-steps was successfully reproduced. In these two adaptation simulations, the main experimental findings of Miles and Kawano (1986) were reproduced only by synaptic plasticity within the cerebellar cortex. Finally, however, if we assumed that the $\mathrm{P}$ cell synaptic plasticity would be induced only when CF inputs preceded the GCA and IC inputs, neither the gain adaptation nor the directional adaptation was reproduced. Based on these simulation results, we discuss the six computational questions raised in the INTRODUCTION.

The first question was whether the model is able to simulate in vivo motor learning with the reported characteristics of the in vitro synaptic plasticity of the cerebellar cortex. The answer was positive for OFR. The success of our simulation suggests that both the acquisition and adaptation of OFR and the drastic transformation of the firing characteristics including the neural codes between the inputs and outputs of the cerebellar cortex all originate from $P$ cell synaptic plasticity. Because there have been no recording-based studies from $\mathrm{P}$ cells during OFR adaptation, we are in the position to make several experimentally testable predictions to examine whether OFR adaptation is really based on cerebellar synaptic plasticity. For the speedstep adaptation, the model predicts specific changes in the CS and SS waveforms and their interactions (see Fig. 9). This could be tested while recording both the CS and SS from a single $\mathrm{P}$ cell during speed-step adaptation. The model also predicts that the ensemble average of the preferred directions of a P cell population will change to the clockwise direction (Fig. 10B) when counterclockwise directional adaptation of eye movements is induced by counterclockwise direction-step stimuli. Accordingly, we propose measuring the average of the directional tuning curves and the average preferred direction from two P-cell populations of equal number before and after the adaptation to examine this prediction.

Regarding the second question, the temporal window for the synaptic plasticity was crucial in reproducing OFR adaptation. The gain and directional adaptations could not be simulated using the temporal window that occurs only after CF modulation, which was derived from the interpretation of Karachot et al. (1994). However, we will show here that their data are consistent with the temporal window that occurs mainly before CF modulation, which appeared in the report by Chen and Thompson (1995). In the study by Karachot et al. (1994), 300 conjunctive stimulations of PFs and CFs were given at 0.25-4 Hz. Figure 11A shows their summary of LTD sizes when the combined stimulation was given at $1 \mathrm{~Hz}$. The horizontal axis represents the delay of $\mathrm{PF}$ activation with respect to $\mathrm{CF}$ activation. The minus signs signify that $\mathrm{PF}$ stimulation preceded $\mathrm{CF}$ stimulation. The plus signs signify that PF stimulation was preceded by $\mathrm{CF}$ stimulation. When the conjunctive stimulations were given cyclically, the shorter one between the PF-CF interval (PF "advance") and the CF-PF interval (PF "delay") seems likely to be the time when LTD occurred. However, they
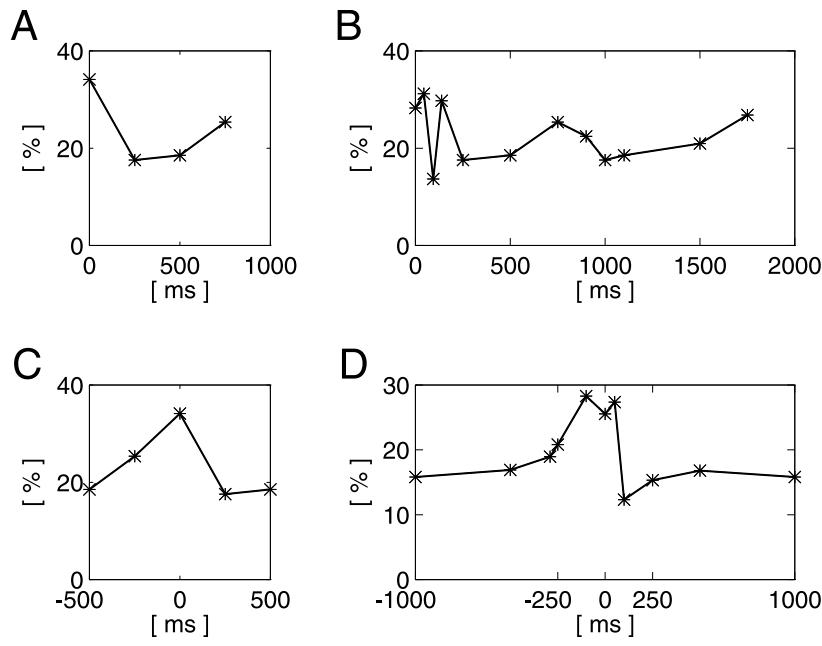

FIG. 11. $A$ and $B$ : long-term depression (LTD) temporal windows reported by Karachot et al. (1994). A: results for combined parallel fibers (PF) and CF stimulation at $1 \mathrm{~Hz}$. The ordinate denotes the average depression of the transmission efficacy $40 \mathrm{~min}$ after the combined stimulation. The abscissa denotes the time delay from $\mathrm{CF}$ stimulus to $\mathrm{PF}$ stimulus. The results were obtained from 300 trials with different delays. $B$ : results of combined stimulations at $0.5-2.0 \mathrm{~Hz}$ plotted in the same way as in $A$. $C$ and $D$ : re-plots of $A$ and $B$ calculating the abscissa as the shorter time interval between the advance of $\mathrm{PF}$ with respect to $\mathrm{CF}$ (negative value) and the delay of $\mathrm{PF}$ with respect to $\mathrm{CF}$ (positive value).

created Fig. $11 \mathrm{~A}$ to represent the delay as the time, even when the delay was longer than the advance. The LTD at the 750-ms delay would be better described as the 250 -ms advance when cyclic stimulation is done at $1 \mathrm{~Hz}$. The data for the $0-, 250-$, 500-, and 750-ms delays in Fig. 11A should be 0, 250, 500, and -500 (delay and advance) and $-250 \mathrm{~ms}$ (advance), respectively (Fig. 11C). Figure $11 B$ is a summary by Karachot et al. (1994) of the LTD sizes collected by conjunctive stimuli at all frequencies, and Fig. $11 D$ is our replot of all of the data shown in Fig. $11 B$ plotted as in Fig. 11C. This replot clearly indicates that LTD was induced maximally during the interval between when the PF inputs preceded the CF inputs by $250 \mathrm{~ms}$ and when the PF inputs followed the CF inputs by $50 \mathrm{~ms}$. This is similar to the temporal window that occurs mainly before CF modulation. We were able to reproduce all of the acquisition and adaptation simulation results with a Gaussian-function temporal window with its peak $100 \mathrm{~ms}$ before the CF inputs with a SD of $50 \mathrm{~ms}$.

The cerebellar motor learning hypothesis (Ito et al. 1982; Kawato 1999) requires that CF inputs carry error information regarding the movements executed. This error information is available only after the movements have been executed and only after the PF inputs have been given to P cells for issuing motor commands. The original interpretation by Karachot et al. (1994) shown in Fig. $11 B$ is at odds with this computational requirement and gives rise to the statement that the LTD of the cerebellar cortex is unable to achieve motor learning (De Schutter 1995; Lukashin 1996). If the two temporal windows reported by Chen and Thompson (1995) and Karachot et al. (1994) are simply added, they cover the period from about 350 ms before CF to $2 \mathrm{~s}$ after CF modulation. Llinas et al. (1997) stated that LTD is unable to function adequately for motor learning with such a long temporal window for synaptic plasticity before and after the $\mathrm{CF}$ inputs. The failure to reproduce OFR experimental data by the temporal window that occurs 
after CF modulation supports this criticism. Consequently, both our reanalysis of the data of Karachot et al. (1994) and our simulation results strongly suggest that the temporal window should be restricted in its width to before CF modulation (e.g., the interval extending from 0 to $300 \mathrm{~ms}$ before $\mathrm{CF}$ modulation), so that LTD is useful for motor learning. However, we should note that differences of $100 \mathrm{~ms}$ in the peak time of the temporal window did not matter much in our simulations as we described before.

The third question was whether or not all reported types of the plasticity, i.e., LTD, LTP, and RP are essential for motor learning (cf. De Schutter 1995). Based on the simulation results, we postulate here that all three are involved in OFR acquisition and learning and that the cerebellum achieves a much more versatile learning capability with these three kinds of plasticity rather than depending on only one kind. For example, LTP is indispensable for acquiring downward eye movements in our model. Without LTP at the v-P cell in Fig. $8 B a$, the GCA inputs with downward preferred directions (Fig. $8 C$, 1st row) would not increase their synaptic efficacy. In other words, the v-P cell could not increase its SS firing with the downward stimulus, and the eyes would not move sufficiently downward. Similarly, LTD and RP are essential for acquiring SS suppression and upward eye movements in response to the upward stimulus (see Fig. 8C, 2nd row).

Inhibitory inputs to $\mathrm{P}$ cells are necessary for the control of OFR. The SS firing frequency increased with the downward stimulus and decreased with the upward stimulus. The spontaneous firing frequency of mossy fibers was almost 0 spikes/s (Kawano and Shidara 1993). With the upward stimulus, the mossy fibers with an upward preferred direction increased their firing frequency, whereas the mossy fibers with a downward preferred direction could not decrease their firing frequency below 0 (because of truncated cosine tuning in the MST and DLPN). This means that, with the upward stimulus, the increased firing frequency of mossy fibers with an upward preferred direction must directly generate SS suppression via inhibitory neurons. Consequently, the OFR control system necessitates inhibitory interneurons within the cerebellar cortex that change the sign of mossy fiber inputs.

Inputs from inhibitory cells to $\mathrm{P}$ cells may also be useful for retaining learning capability in the adult state. In our model, the absence of SS modulation in a P cell does not mean that there is no modulation in GCA or IC inputs to that $\mathrm{P}$ cell. For example, as shown in Fig. $8 B a$, when leftward visual motion was given to the left v-P cell, SS was not modulated, but the leftward GCA and IC inputs were maximally activated and exerted their excitatory and inhibitory effects on the left $\mathrm{v}-\mathrm{P}$ cell. However, their actions canceled out, because the synaptic strengths were balanced between excitation and inhibition. At first glance, these synapses appear useless and wasteful, but they are eventually used efficiently in subsequent directionalstep adaptations, for example, as shown in the 2 nd row of Fig. $10 C$ (LTP on excitatory synapses). Therefore the existence of inhibitory synapses and their synaptic plasticity (RP) enable nonzero excitatory synaptic weights to survive without inducing unnecessary SS modulations. This dynamic balance between excitatory and inhibitory synaptic actions in the adultstate equilibrium endows the OFR system with a versatile adaptive capability for preparing for future unpredictable environments, such as direction-step stimuli.
If there are no synapses on v-P cells from GCAs and ICs with preferred horizontal directions, the horizontal preferred inputs should construct their synapses from "zero" when direction-step stimuli come. The synaptic weights of the verticalpreferring GCA and IC inputs on v-P cells would not be zero at the beginning because $\mathrm{V}-\mathrm{P}$ cells modulate their firing with vertical stimuli. Thus the weight of vertical-preferred synapses would increase or decrease from a nonzero point during vertical gain adaptations. The synaptic construction from zero that might appear in directional adaptation seems to take longer than the changes from nonzero that might appear in gain adaptations (synaptic sprouting vs. synaptic plasticity). Monkeys do not show slower adaptation for direction adaptation than gain adaptation (Miles and Kawano 1986), which might mean that the synaptic change in directional adaptation is not from the zero point but from a nonzero point as the present model hypothesized.

Regarding the fourth question, our model was able to simulate the acquisition of OFR as well as the attendant gains and orientation adaptations. If $\mathrm{P}$ cells are provided with a rich set of GCA and IC inputs and the appropriate error signals via $\mathrm{CF}$ inputs, the cerebellum can achieve a wide variety of movement adaptations.

The simulated two-dimensional trajectory of eye velocity for the four-direction test ramp stimuli changed from the beginning of the movement after repeated direction-step stimuli (Fig. 10Ac). For example, the initial trajectory of the left stimulus was simulated as being in the left-downward direction. On the other hand, Miles and Kawano (1986) reported that the adapted trajectory for the leftward ramp stimulus was initially leftward and that it gradually changed to downward. The difference between our simulation and empirical data may come from the model's lack of temporal firing delays, which GCAs' and ICs' inputs might possess. We modeled the delay between visual input and the firing of GCAs and ICs using a single value of $39 \mathrm{~ms}$ for all inputs. Meanwhile, individual GCAs and ICs might have individual delays. The recorded firings were for the cells that responded mainly before the eye movements (Kawano et al. 1992, 1994). Some cells in the MST and DLPN may respond even later. Other delays might be constructed in the neural network of the cerebellar granular layer, which contains many granular cells and Golgi cells. A model with such delays might reproduce a similar adapted trajectory reported by Miles and Kawano (1986).

The fifth question was what proportion of the SS firing characteristics of an individual $\mathrm{P}$ cell are determined by the characteristics of the $\mathrm{CF}$ inputs to that $\mathrm{P}$ cell through synaptic plasticity. In our simulation, we assumed that the GCA and IC synaptic weights were small random values in the "inborn state." We also assumed that only CFs with the upward preferred direction could innervate vertical $P$ cells and that only CFs with the contralateral preferred direction could innervate horizontal $\mathrm{P}$ cells at birth. These assumptions were natural because the accessory optic system, which is older than the cortical visual system in evolutionary terms, already possesses upward preferred direction (e.g., lateral terminal nuclei) and ipsilateral preferred directions (note: the nucleus of the optic tract projects first to the inferior olive and then to the contralateral VPFL) (Fuchs and Mustari 1993; Mustari et al. 1994), whereas the MST and DLPN have almost uniformly distributed preferred directions. The genetic information could be more 
precise for the CF innervations that ultimately come from the accessory optic system than the GCA and IC innervations that originate in MST and DLPN. Another behavioral observation that supports our assumption about random GCA/IC synaptic weights is that there has been no report of the existence of OFR in newborn monkeys or humans as far as we know. Furthermore, one study has shown that OFR does not exist in a 10-wk-old baby (R. Osu, personal communication). Under these assumptions, the SS firing characteristics after OFR acquisition are mostly determined by the $\mathrm{CF}$ inputs to the $\mathrm{P}$ cell, for directional tuning, speed tuning, modulation amplitude, and the temporal waveform of the firing frequency. In this scenario, the mossy fiber input system only delineates the possible repertoire of individual $\mathrm{P}$ cells, and the $\mathrm{CF}$ input determines the ultimate SS characteristics through motor learning. One explicit experimental use of our model in studying the learning of OFR in infants might be to record SSs and CSs of infant monkeys before they acquire OFR. CSs may have preferred upward or contralateral directions, whereas SSs might not modulate much or have preferred directions.

The last question was about what the cerebellar cortex computationally acquires during motor learning. Kawato et al. (1987) postulated that the cerebellum acquires an inverse dynamics model of the motor apparatus through the feedbackerror-learning scheme. The feedback-error-learning scheme is a two-degree-of-freedom control system that consists of a negative feedback loop and a feed-forward control pathway. The inverse dynamics model in the feed-forward path is acquired through learning, while it uses the feedback motor command computed in the crude feedback loop as the error signal. Some concrete examples of cerebellar learning have been formulated in this framework of feedback-error learning (Kawato and Gomi 1992). In particular, the OFR system was interpreted in the following way (Kawato 1999). The VPFL constitutes the inverse model. The CF input carries the feedback motor command, which can be used as the error signal for motor commands. This motor-command error signal originates in the accessory optic system, which is a crude feedback controller. Previous experimental OFR studies (Gomi et al. 1998; Kobayashi et al. 1998; Shidara et al. 1993; Takemura et al. 2001) have already provided support for some of these assumptions. The cerebellar feedback-error-learning theory was much more concretely and quantitatively formulated for the OFR in this paper and could be more directly tested in future physiological experiments.

In the model with small, random synaptic weights in the inborn state, the SSs of P cells were not well modulated (Fig. $5 C$ ), and the eyes did not move sufficiently to follow the visual stimulus (Fig. 5D). After learning, however, the SS was well modulated by the visual stimulus (Fig. $5 E$ ), and the eyes moved sufficiently (Fig. 5D). Before learning, the coefficients for the reconstruction of simulated SSs by the acceleration, velocity, and position of eye movements were 0,0 , and 0 , respectively, whereas they were $0.112,2.61$, and -6.97 after learning. Because we assumed that the oculomotor plants were secondorder plants, the VPFL acquires the corresponding second order inverse models of the eye movements that are necessary for compensating for dynamic forces for the second order plant. In summary, the cerebellar feedback-error-learning theory has postulated that inverse models are acquired in the cerebellum by learning. We have presented detailed simulation studies supporting this theory based on intensive and extensive neurophysiological data obtained for OFR.

We thank Drs. H. Gomi, K. Doya, S. Kitazawa, and Y. Inoue for fruitful discussions, Dr. F. A. Miles for useful suggestions, M. Namba for administrative assistance, and Prof. T. Bando for encouragement.

\section{REFERENCES}

BAIZER JS AND GLICKSTEIN M. Role of cerebellum in prism adaptation. J Physiol (Lond) 236: 34-35, 1974.

Baizer JS, Kralj-HANS I, AND GLiCKSTEIN M. Cerebellar lesion and prism adaptation in macaque monkeys. J Neurophysiol 81: 1960-1965, 1999.

Boussaoud D, Desimone R, AND UnGERLEIDER LG. Subcortical connections of visual areas MST and FST in macaques. Vis Neurosci 9: 291-302, 1992.

BRodal P. The corticopontine projection in the rhesus monkey: origin and principles of organization. Brain 101: 251-283, 1978.

Chen C And Thompson RF. Temporal specificity of long-term depression in parallel fiber-Purkinje synapses in rat cerebellar slice. Learn Mem 2: 185$198,1995$.

Crepel F, Hemart N, Jaillard D, and Daniel H. Cellular mechanisms of long-term depression in the cerebellum. Behav Brain Sci 19: 347-353, 1996.

DE SCHutTer E. Cerebellar long-term depression might normalize excitation of Purkinje cells: a hypothesis. Trends Neurosci 18: 291-295, 1995.

De Zeeuw CI, Hansel C, Bian F, KoekKoek SKE, van Alphen AM, Linden DJ, AND OBERDICK J. Expression of a protein kinase $\mathrm{C}$ inhibitor in Purkinje cells blocks cerebellar LTD and adaptation of the vestibulo-ocular reflex. Neuron 20: 495-508, 1998.

Dufosse M, Ito M, Jastreboff PJ, And Miyashita Y. Neuronal correlate in rabbit's cerebellum to adaptive modification of the vestibulo-ocular reflex. Brain Res 150: 611-616, 1978.

Fuchs AF AND Mustari MJ. The optokinetic response in primates and its possible neuronal substrate. In: Visual Motion and its Role in the Stabilization of Gaze. Amsterdam: Elsevier, 1993, p. 343-369.

FuJITA M. Simulation of adaptive modification of the vestibulo-ocular reflex with an adaptive filter model of the cerebellum. Biol Cybern 45: 207-214, 1982.

FukUShima K, KANEKo CRS, AND FuCHS AF. The neuronal substrate of integration in the oculomotor system. Prog Neurobiol 39: 609-639, 1992.

Gauthier GM, Hofferer JM, Hoyt WF, AND Stark L. Visual-motor adaptation: quantitative demonstration in patients with posterior fossa involvement. Arch Neurol 36: 155-160, 1979.

Gellman RS, CARL JR, AND Miles FA. Short latency ocular-following responses in man. Vis Neurosci 5: 107-122, 1990.

GILBERT PFC AND THACH WT. Purkinje cell activity during motor learning. Brain Res 128: 309-328, 1977.

Glickstein M, Cohen JL, Dixon B, Gibson A, Hollins M, Lanossiere E, AND RoBinson F. Corticopontine visual projections in macaque monkeys. J Comp Neurol 190: 209-229, 1980.

Glickstein M, May J, And Mercer BE. Corticopontine projection in the macaque: the distribution of labeled cortical cells after large injections of horse-radish peroxidase in the pontine nuclei. J Comp Neurol 235: 343-359, 1985 .

Glickstein M, Mersier B, Stein J, LegG C, And Voogd J. Visual pontine projection to the cerebellar hemispheres of macaques. Soc Neurosci Abstr 16: $637,1990$.

Gomi H And Kawato M. Adaptive feedback control models of the vestibulocerebellum and spinocerebellum. Biol Cybern 68: 105-114, 1992.

Gomi H, Shidara M, Takemura A, Inoue Y, Kitama H, Kawano K, and KawATO M. Temporal firing patterns of Purkinje cells in the cerebellar ventral paraflocculus during ocular following responses in monkeys. I. Simple spike. J Neurophysiol 80: 818-831, 1998.

HiRANO T. Depression and potentiation of the synaptic transmission between a granule cell and a Purkinje cell in rat cerebellar culture. Neurosci Lett 119: 141-144, 1990.

InOUE Y, TAKemura A, Kawano K, And Mustari MJ. Role of the pretectal nucleus of the optic tract in short-latency ocular following responses in monkeys. Exp Brain Res 131: 269-281, 2000.

Ito M, JASTREBOFF PJ, AND MiYAShita Y. Adaptive modification of the rabbit's horizontal vestibulo-ocular reflex during sustained vestibular and optokinetic stimulation. Exp Brain Res 37: 17-30, 1979.

Ito M AND Kano M. Long-lasting depression of parallel fiber-Purkinje cell transmission induced by conjunctive stimulation of parallel fibers and climbing fibers in the cerebellar cortex. Neurosci Lett 33: 253-258, 1982. 
Ito M, Sakurai M, AND Tongroach P. Climbing fiber induced depression of both mossy fibre responsiveness and glutamate sensitivity of cerebellar Purkinje cells. J Physiol (Lond) 324: 113-134, 1982.

KANO M. Long-lasting potentiation of GABAergic inhibitory synaptic transmission in cerebellar Purkinje cells: its properties and possible mechanisms. Behav Brain Sci 19: 354-361, 1996.

Kano M, Rexhausen U, Dreessen J, and Konnerth A. Synaptic excitation produces a long-lasting rebound potentiation of inhibitory synaptic signals in cerebellar Purkinje cells. Nature 356: 601-604, 1992.

KARACHOT L, KADO RT, AND ITO M. Stimulus parameters for induction of long-term depression in in-vitro rat Purkinje cells. Neurosci Res 21: 161$168,1994$.

KAWANO K. Ocular tracking: behavior and neurophysiology. Curr Opin Neurobiol 9: 467-473, 1999.

KAWANO K AND SHIDARA M. The role of the ventral paraflocculus in ocular following in the monkey. In: Role of the Cerebellum and Basal Ganglia in Voluntary Movement. Amsterdam: Elsevier, 1993, p. 195-202.

Kawano K, Shidara M, and Yamane S. Neural activity in dorsolateral pontine nucleus of alert monkey during ocular following responses. $\mathrm{J} \mathrm{Neu-}$ rophysiol 67: 680-703, 1992.

Kawano K, Shidara M, Watanabe Y, and Yamane S. Neural activity in cortical area of MST of alert monkey during ocular following responses. J Neurophysiol 71: 2305-2324, 1994.

KAWATO M. Internal models for motor control and trajectory planning. Curr Opin Neurobiol 9: 718-727, 1999.

Kawato M, Furukawa K, and SuzuKi R. A hierarchical neural-network model for control and learning of voluntary movement. Biol Cybern 57: 169-185, 1987.

Kawato M AND Gomi H. A computational model of four regions of the cerebellum based on feedback-error-learning. Biol Cybern 68: 95-103, 1992.

Kettner RE, Mahamud S, Leung HC, Sitokoff N, Houk JC, and Peterson BW. Prediction of complex two-dimensional trajectories by a cerebellar model of smooth pursuit eye movement. J Neurophysiol 77: 2115-2130, 1997.

KitaZawa S, Kimura T, and Yin PB. Cerebellar complex spikes encode both destinations and errors in arm movements. Nature 392: 494-497, 1998.

Kobayashi Y, Kawano K, Takemura A, Inoue Y, Kitama T, Gomi H, and Kawato M. Temporal firing patterns of Purkinje cells in the cerebellar ventral paraflocculus during ocular following responses in monkeys. II. Complex spikes. J Neurophysiol 80: 832-848, 1998.

Langer T, Fuchs AF, Scudder CA, and Chubb MC. Afferents to the flocculus of the cerebellum in the Rhesus Macaque as revealed by retrograde transport of horseradish peroxidase. J Comp Neurol 235: 1-25, 1985.

Li J, Smith SS, AND McElligott JG. Cerebellar nitric oxide is necessary for vestibuloocular reflex adaptation, a sensorimotor model of learning. J Neurophysiol 74: 489-494, 1995.

Llinas R, LANG EJ, AND Welsh JP. The cerebellum, LTD, and memory: alternative views. Learn Mem 3: 445-455, 1997.

Lisberger SG, Miles FA, And ZeE DS. Signals used to compute errors in monkey vestibulo-ocular reflex: possible role of flocculus. J Neurophysiol 52: $1140-1153,1984$.

LUKASHIN AV. Cortical dynamic tuning: the role of intrinsic connections, as revealed by modeling. Curr Opin Neurobiol 6: 765-772, 1996.

MAUNSELl JHR AND VAN EsSEN DC. The connections of the middle temporal visual area (MT) and their relationship to a cortical hierarchy in the macaque monkey. J Neurosci 3: 2563-2586, 1983.

MAY JG AND ANDERSEN RA. Different patterns of corticopontine projections from separate cortical fields within the inferior parietal lobule and dorsal prelunate gyrus of the macaque. Exp Brain Res 63: 265-278, 1986.

McElligott JG, BeEton P, AND PolK J. Effect of cerebellar inactivation by lidocaine microdialysis on the vestibuloocular reflex in goldfish. $J$ Neurophysiol 79: 1286-1294, 1998.

MichNOVICZ JJ AND BENNETT MVI. Effects of rapid cerebellectomy on adaptive gain control of the vestibulo-ocular and optokinetic responses in pigmented rabbits. Exp Brain Res 66: 287-294, 1987.
Miles FA and Kawano K. Short-latency ocular following responses of monkey. III. Plasticity. J Neurophysiol 56: 1381-1396, 1986.

Miles FA, Kawano K, and Optican LM. Short-latency ocular following responses of monkey. I. Dependence of temporospatial properties of visual input. J Neurophysiol 56: 1321-1354, 1986.

Mustari MJ, Fuchs AF, Kaneko CR, And Robinson FR. Anatomical connections of the primate pretectal nucleus of the optic tract. J Comp Neurol 349: 111-128, 1994.

NAGAO S. Effects of vestibulocerebellar lesions upon dynamic characteristics and adaptation of vestibulo-ocular and optokinetic responses in pigmented rabbits. Exp Brain Res 53: 36-46, 1983.

NAGAO S. Behavior of floccular Purkinje cells correlated with adaptation of vestibulo-ocular reflex in pigmented rabbits. Exp Brain Res 77: 531-540, 1989.

NAGaO S AND Ito M. Subdural application of hemoglobin to the cerebellum blocks vestibulo-ocular reflex adaptation. Neuroreport 2: 193-196, 1991.

PAStor AM, De Cruz RR, AND BAKER R. Cerebellar role in adaptation of the goldfish vestibuloocular reflex. J Neurophysiol 72: 1383-1394, 1994.

RAYMOND JL AND LISBERGER SG. Neural learning rules for the vestibulo-ocular reflex. J Neurosci 18: 9112-9129, 1998.

RoBINSON DA. Adaptive gain control of vestibuloocular reflex by the cerebellum. J Neurophysiol 39: 954-969, 1976.

SAKURAI M. Synaptic modification of parallel fibre-Purkinje cell transmission in in-vitro guinea-pig cerebellar slices. J Physiol (Lond) 394: 463-480, 1987.

Sanes JN, Dimitrov B, and Hallett M. Motor learning in patients with cerebellar dysfunction. Brain 113: 103-120, 1990

SCHWEIGHofer N, ARBiB MA, AND Dominey PF. A model of the cerebellum in adaptive control of saccadic gain. II. Simulation results. Biol Cybern 75: 29-36, 1996.

Schweighofer N, Spoelstra J, Arbib MA, and Kawato M. Role of the cerebellum in reaching movements in humans. II. A neural model of the intermediate cerebellum. Eur J Neurosci 10: 95-105, 1998.

ShidARA K AND Kawano K. Role of Purkinje cells in the ventral paraflocculus in short-latency ocular following responses. Exp Brain Res 93: 185-195, 1993.

Shidara M, Kawano K, Gomi H, and Kawato M. Inverse-dynamics encoding of eye movements of Purkinje cells in the cerebellum. Nature 365: $50-52,1993$.

Takemura A, Inoue $\mathrm{Y}$, Gomi $\mathrm{H}$, Kawato $\mathrm{M}$, and Kawano K. Change in neuronal firing patterns in the process of motor command generation for the ocular following response. J Neurophysiol 86: 1750-1763, 2001.

Thach WT, Goodkin HP, AND Keating JG. The cerebellum and the adaptive coordination of movement. Аnпи Rev Neurosci 15: 403-442, 1992.

TUSA RJ AND UNGERLEIDER LG. Fiber pathways of cortical areas mediating smooth pursuit eye movements in monkeys. Ann Neurol 23: 174-183, 1988.

Ungerleider LG, Desimone R, GaLkin TW, AND Mishrin M. Subcortical projections of area MT in the macaque. J Comp Neurol 223: 368-386, 1984.

WATANABE E. Role of the primate flocculus in adaptation of the vestibuloocular reflex. Neurosci Res 3: 20-38, 1985.

Weiner MM, Hallett M, AND FunKenstein HH. Adaptation to lateral displacement of vision in patients with lesions of the central nervous system. Neurology 33: 766-772, 1983.

YANAGIHARA D AND KoNDO I. Nitric oxide plays a key role in adaptive control of locomotion in cat. Proc Natl Acad Sci USA 93: 13292-13297, 1996.

Yamamoto K, Kobayashi Y, Takemura A, Kawano K, and Kawato M. A mathematical model that reproduces vertical ocular following responses from visual stimuli by reproducing the simple spike firing frequency of Purkinje cells in the cerebellum. Neurosci Res 29: 161-169, 1997.

Yamamoto K, Kobayashi Y, Takemura A, Kawano K, and Kawato M. A mathematical analysis of the characteristics of the system connecting the cerebellar ventral paraflocculus and extraoculomotor nucleus of alert monkeys during upward ocular following responses. Neurosci Res 38: 425-435, 2000 . 